Manuscript Number: AB-19-2920R2

Title: Multicomponent hydrogels for the formation of vascularized bonelike constructs in vitro

Article Type: Full length article

Keywords: Self-assembly; peptide nanofiber; 3D cell culture; angiogenesis; bone tissue engineering

Corresponding Author: Professor Alvaro Mata,

Corresponding Author's Institution: Queen Mary, University of London

First Author: Burak Derkus, PhD

Order of Authors: Burak Derkus, PhD; Babatunde O Okesola, PhD; David W Barrett, PhD; Matteo D'Este, PhD; Tina T Chowdhury, PhD; David Eglin, PhD; Alvaro Mata, PhD

Abstract: The native extracellular matrix (ECM) is a complex gel-like system with a broad range of structural features and biomolecular signals. Hydrogel platforms that can recapitulate the complexity and signaling properties of this ECM would have enormous impact in fields ranging from tissue engineering to drug discovery. Here, we report on the design, synthesis, and proof-of-concept validation of a microporous and nanofibrous hydrogel exhibiting multiple bioactive epitopes designed to recreate key features of the bone ECM. The material platform integrates self-assembly with orthogonal enzymatic cross-linking to create a supramolecular environment comprising hyaluronic acid modified with tyramine (HA-Tyr) and peptides amphiphiles (PAs) designed to promote cell adhesion (RGDS-PA), osteogenesis (Osteo-PA), and angiogenesis (Angio-PA). Through individual and co-cultures of human adipose derived mesenchymal stem cells (hAMSCs) and human umbilical vascular endothelial cells (HUVECs), we confirmed the capacity of the HA-Tyr/RGDS-PA/Osteo-PA/AngioPA hydrogel to promote cell adhesion as well as osteogenic and angiogenic differentiation in both 2D and 3D setups. Furthermore, using immunofluorescent staining and reverse transcription-quantitative polymerase chain reaction (RT-qPCR), we demonstrated co-differentiation and organization of hAMSCs and HUVECs into 3D aggregates resembling vascularized bone-like constructs. 


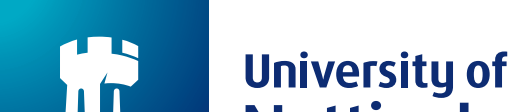 Nottingham \\ UK | CHINA | MALAYSIA}

University of Nottingham

University Park

Nottingham

NG7 2RD

March $13^{\text {th }}, 2020$

Dr. Marc Bohner

Editor

Acta Biomaterialia

Dear Dr. Bohner,

Thank you again for the opportunity to improve and resubmit our work titled "Multicomponent Hydrogels for the Formation of Vascularized Bone-like Constructs In Vitro".

We have addressed both comments from the reviewer and modified the manuscript accordingly (with green highlights). We have done this also for the second comment, which requests further experiments. However, we respectfully disagree with the reviewer and have elaborated our rationale in the Response to Reviewers document.

We hope that you will agree that this modified version of the manuscript satisfactorily addresses all the reviewers' concerns and demonstrates the potential of our approach.

Thank you in advance for your consideration and we look forward to your response.

Sincerely,

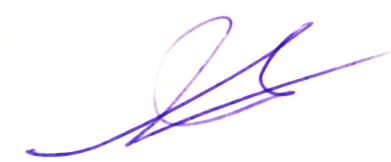

\section{Alvaro Mata}

Chair in Biomedical Engineering and Biomaterials

School of Pharmacy, Division of Regenerative Medicine and Cellular Therapies

Department of Chemical \& Environmental Engineering 


\section{Statement of Significance}

This body of work presents a new approach to develop more complex, yet functional, in vitro environments for cell culture while enabling a high level of control, tuneability, and reproducibility. The multicomponent self-assembling bioactive $2 \mathrm{D}$ and 3D hydrogels with nanofibrous architecture designed to recreate key molecular and macromolecular features of the native bone ECM and promote both osteogenesis and angiogenesis. The materials induce endothelial cells towards large vascular lumens and MSCs into bone cells on/within the same platform and form vascularized-bone like construct in vitro. This strategy looks encouraging for lifelike bone tissue engineering in vitro and bone tissue regeneration in vivo. 


\section{Response to Reviewers}

1. Information regarding reproducibility are still missing: How many repetitions of the experiments? Especially, the authors used only one cell line.

We thank the reviewer for pointing this out. We have now added the number of repeats (highlighted in green) for all the experiments conducted including: the zeta potential (page 5), degradation (page 6), cell viability (page 7), cell proliferation (page 7), angiogenesis (page 7), confocal investigations of co-culture (page 8), and quantification of gene expression of Runx 2 (for osteogenic differentiation and co-culture study) as well as CD31 and VEGF (for angiogenesis and co-culture study) in 2D and 3D conditions (page 8).

2. Regarding the characterization of the gel seeded with hAMSC, the authors relied on a combination of qPRC and immunostaining to demonstrate osteogenetic differentiation, which is ok. To proof the osteogenic differentiation, I would recommend to show at least three markers for each method.

We understand that more experiments can be performed to further characterize different parts of the study. However, we disagree that they are needed and would like to take the opportunity to elaborate on this rationale.

First, our study encompasses a broad spectrum of work including material design, characterization, optimization, and biological validation. It also reports on a multifunctional material with enhanced molecular complexity and increased number of functions to validate. In this context, our study aims to demonstrate proof-of-concept of this multifunctionality rather than conduct an in-depth characterization of every variable. Our goal is to demonstrate the potential of our design and inspire studies that address this need for material complexity.

However, we have carefully designed our experiments to obtain sufficient data, which is in accordance with many previous studies. For example, previous studies have demonstrated osteoinductive properties of new materials based solely on gene expression data of one (Tuin et al., Acta Biomaterialia 2016; Fraioli et al., Acta Biomaterialia 2016) or two (Heras et al., Acta Biomaterialia 2019) markers, or a combination of gene expression and immunofluorescence (Moore et al., Acta Biomaterialia 2011). In our study, we have conducted immunofluorescence investigations on Runx2, ALP, and Osn as well as gene expression data for Runx2.

We hope that reviewer agrees that the data we have conducted is sufficient to demonstrate proof-of-concept. To make this clearer, we have added text (highlighted in green) on pages 2 and 15.

\section{References}

1. Tuin et al. Fabrication of novel high surface area mushroom gilled fibers and their effects on human adipose derived stem cells under pulsatile fluid flow for tissue engineering applications. Acta Biomater. 2016;36:220-30

2. Heras et al. Osteostatin potentiates the bioactivity of mesoporous glass scaffolds containing $\mathrm{Zn}^{2+}$ ions in human mesenchymal stem cells. Acta Biomater. 2019;15;89:359-371.

3. Moore et al. Synergistic enhancement of human bone marrow stromal cell proliferation and osteogenic differentiation on BMP-2-derived and RGD peptide concentration gradients. Acta Biomater. 2011;7(5):2091-100.

4. Fraioli et al. Surface guidance of stem cell behavior: Chemically tailored copresentation of integrin-binding peptides stimulates osteogenic differentiation in vitro and bone formation in vivo. Acta Biomater. 2016 Oct 1;43:269-281. 

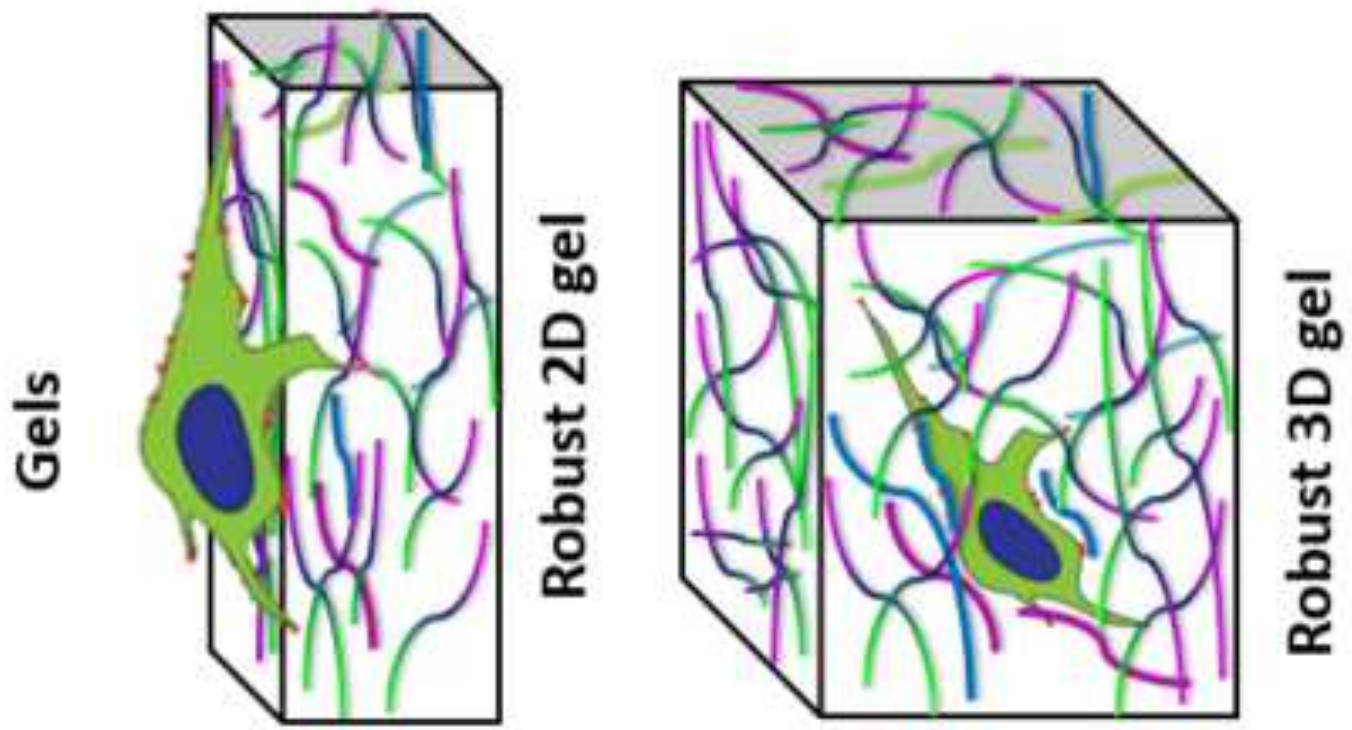

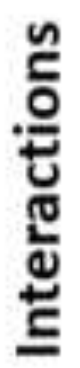
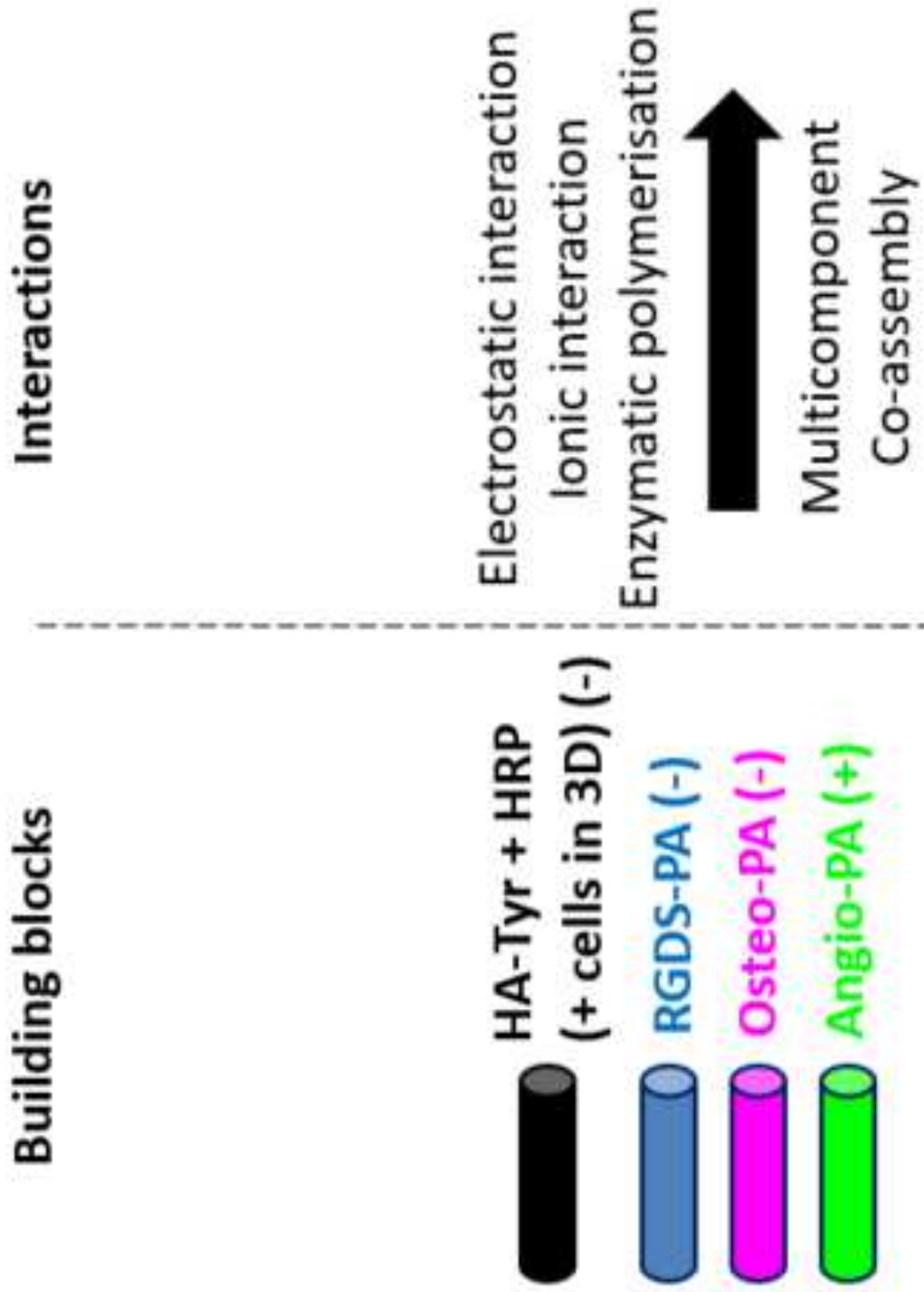


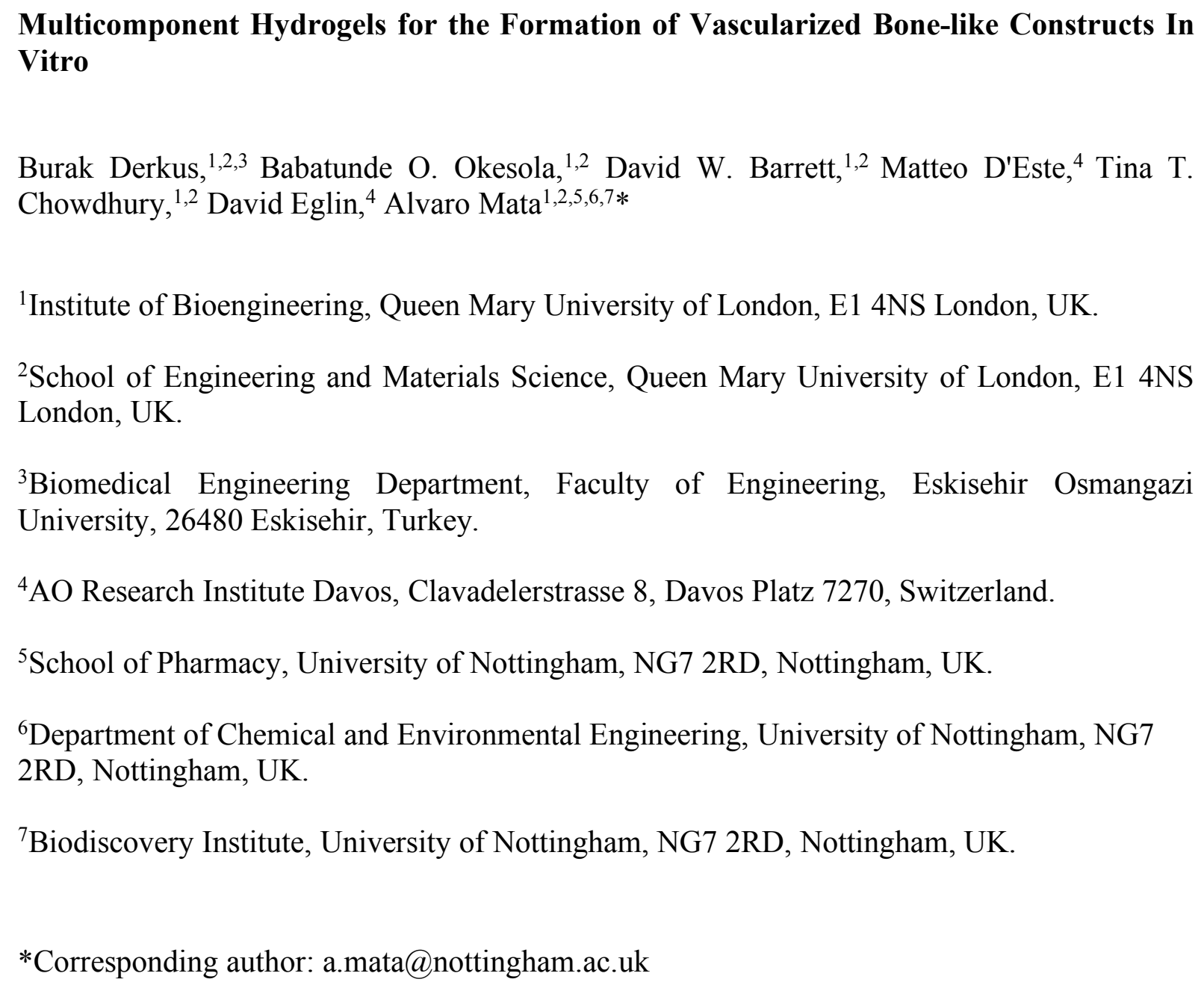




\begin{abstract}
1 Abstract
2 The native extracellular matrix (ECM) is a complex gel-like system with a broad range of 3 structural features and biomolecular signals. Hydrogel platforms that can recapitulate the 4 complexity and signaling properties of this ECM would have enormous impact in fields ranging 5 from tissue engineering to drug discovery. Here, we report on the design, synthesis, and proof6 of-concept validation of a microporous and nanofibrous hydrogel exhibiting multiple bioactive 7 epitopes designed to recreate key features of the bone ECM. The material platform integrates 8 self-assembly with orthogonal enzymatic cross-linking to create a supramolecular environment 9 comprising hyaluronic acid modified with tyramine (HA-Tyr) and peptides amphiphiles (PAs) 10 designed to promote cell adhesion (RGDS-PA), osteogenesis (Osteo-PA), and angiogenesis 11 (Angio-PA). Through individual and co-cultures of human adipose derived mesenchymal stem 12 cells (hAMSCs) and human umbilical vascular endothelial cells (HUVECs), we confirmed the 13 capacity of the HA-Tyr/RGDS-PA/Osteo-PA/Angio-PA hydrogel to promote cell adhesion as 14 well as osteogenic and angiogenic differentiation in both 2D and 3D setups. Furthermore, using 15 immunofluorescent staining and reverse transcription-quantitative polymerase chain reaction 16 (RT-qPCR), we demonstrated co-differentiation and organization of hAMSCs and HUVECs 17 into 3D aggregates resembling vascularized bone-like constructs.
\end{abstract}

18 Keywords: Self-assembly, peptide nanofiber, 3D cell culture, angiogenesis, bone tissue 19 engineering 


\section{Introduction}

Bone is a complex tissue comprising hierarchically organized and mineralized collagen fibers, vasculature, and numerous specialized cells. Its architecture consists of a range of fibrous and porous structures with varying degrees of density, stiffness, and organization expanding the nano- (e.g. mineralized fibers), sub-micro- (e.g. lamella), and micro- (e.g. osteons) scales. This structure, and consequently its resulting properties, emerge from a rich organic matrix including primarily collagens, bone sialoproteins, osteocalcin, and proteoglycans, which additionally regulate cell metabolism and direct cell behaviors. Given the functionality of this matrix, there is increasing interest to recreate it in order to engineer bio-inspired 3D materials with precise biomolecular, chemical, and mechanical properties.

A plethora of bone biomaterial approaches are being explored that expand from reversible [1] and supramolecular chemistry [2] to decellularized tissues [3] and biofabrication [4]. Examples include hyper-elastic calcium phosphates [4], bioactive [5], and self-healing [6] gels and membranes [7,8], injectable nanogels [9], and growth factor releasing gels [10]. Selfassembling peptides offer an attractive avenue to recreate molecular and physical features of the native extracellular matrix (ECM) [11]. Peptide amphiphiles (PAs) have emerged as a leading self-assembling platform to engineer matrices by design and with molecular precision [12]. PAs consist of a hydrophobic tail that drives self-assembly, a $\beta$-sheet forming amino acid sequence that stabilizes the assembled nanofibres, and a charged functional head group that facilitates solubility in aqueous environments. These molecules can present bioactive epitopes to stimulate cell behaviors such as adhesion [13], migration [14], and differentiation [15] as well as biological processes such as mineralization [5]. However, recreation of the complexity, diversity, and dynamic nature of the ECM remains an unmet challenge.

New approaches are being explored to enhance the functionality of PAs such as enabling responsive behaviors [16] or guiding interactions with other functional biomolecules [17]. Multicomponent self-assembly offers an opportunity to not only exploit the inherent benefits of different individual building-blocks but also synergistic effects as a result of their interaction $[18,19]$. Through this strategy, PAs have been co-assembled with drugs to enhance tumortargeting [20], polysaccharides to grow hierarchical membranes and capsules [21], proteins to engineer complex hierarchical scaffolds [22], or DNA to generate peptide-DNA superstructures [23] or nanowires [24]. These approaches are enabling the capacity to build more complex systems that can more accurately recreate specific biological scenarios.

An ideal matrix designed to recreate bone tissue must primarily promote the growth of boneforming cells such as osteoblasts and endothelial cells. Towards this goal, bioactive epitopes have been incorporated into self-assembling materials. For example, the commercially available RADA-I peptide was used to induce mesenchymal stem cells (MSCs) towards an osteogenic lineage [25] while PA molecules bearing the Collagen-I mimetic peptide DGEA were shown to promote adhesion and osteogenic differentiation of MSCs [26]. Moreover, the combination of DGEA and the cell adhesion ligand RGDS has been reported to promote osteogenic differentiation [27]. On the other hand, peptides mimicking segments of vascular 
endothelial growth factor (VEGF) such as QK [28], KLTWQELYQLKYKGI [29], and K2(SL)6K2 [30] or the osteopontin-derived peptide SVVYGLR have been shown to have angio-inductive properties both in vitro [31] and in vivo [32]. Taking advantage of the versatility of PAs and multicomponent self-assembly, it may be possible to design more complex multifunctional ECM-like matrices that present a number of bioactive epitopes as well as key ECM macromolecules.

In this study, we report a multicomponent self-assembling 3D hydrogel designed to recreate key molecular and macromolecular features of the native bone ECM and promote both osteogenesis and angiogenesis. The material is formed by co-assembling HA with PAs bearing different bioactive epitopes and taking advantage of both covalent and non-covalent interactions. We used HA to provide a biocompatible and pro-angiogenic ECM macromolecule [33], which was functionalized with Tyr (HA-Tyr) to control stiffness and stability through enzyme-mediated oxidative coupling as described by Eglin and co-workers [34]. The PAs were designed to promote cell adhesion (RGDS-PA: C16-V3A3E3E3RGDS), endothelial vascular organization (Angio-PA: C16-V3A3K3SVVYGLR), and osteoblastic differentiation (OsteoPA: C16-V3E3DGEA) (Figure 1a). Previous studies have reported on the interfacial formation of co-assembling membranes between a solution of HA and a solution of oppositely charged self-assembling peptides [21]. Here, to enable the formation of 2D/3D environments, HA-Tyr was first mixed with the negatively charged Osteo-PA and RGDS-PA and then mixed with the positively charged Angio-PA to trigger co-assembly and gel formation (Figure 1b). The system opens opportunities for the development of more complex and biologically relevant in vitro models of bone tissue as well as materials for bone regeneration.

\section{Materials and Methods}

\subsection{Synthesis and purification of $\beta$-sheet forming $P A s$}

PAs at $1 \mathrm{mmol}$ scale were synthesized using a solid-phase peptide synthesis technique based on the 9-fluorenylmethoxycarbonyl (Fmoc) protection chemistry on an automated peptide synthesizer (Liberty Blue, CEM, USA). Additional notes on this method can be found in Supplementary Information (SI). The synthesized PAs were purified via Reverse Phase-High Performance Liquid Chromatography/Mass Spectroscopy (RP-HPLC/MS) and characterized with Electrospray Ionization-Mass Spectroscopy (ESI-MS) to confirm the molecular weight (the spectra have been presented in SI).

\subsection{Characterization of PAs with Zeta Sizer, Circular Dichroism (CD), and Transmission Electron Microscopy (TEM)}

Zeta potentials $(\zeta)$ were measured to determine the overall charges of Angio-PA, RGDS-PA, and Osteo-PA molecules under specific $\mathrm{pH}$ at $25{ }^{\circ} \mathrm{C}$ on a Zetasizer (MPT-2 Instrument, Malvern Panalytical, UK). PAs were individually dissolved $(0.1 \%)$ in HEPES(4-(2hydroxyethyl)-1-piperazineethanesulfonic acid) buffer with sodium chloride ( $\mathrm{NaCl}, 0.9 \%), \mathrm{pH}$ was adjusted to 7.4 with ammonium hydroxide $\left(\mathrm{NH}_{4} \mathrm{OH}, 0.1 \mathrm{M}\right)$, and the solutions were 
transferred into cuvettes equipped with electrodes for zeta potential measurement. Each measurement was repeated three times.

For the conformational identification of the designed PAs, CD spectra were obtained for PAs at $\mathrm{pH} 7.4$ and $25^{\circ} \mathrm{C}$. Aqueous solutions of PA molecules $(0.1$ wt.) were loaded into quartz cuvettes with $1 \mathrm{~cm}$ path length and spectra were acquired on $\mathrm{CD}$ spectrometer (Applied Photophysics Limited, UK). Measurements were performed within the range of 180-260 nm. The spectra were obtained for an average of five accumulations acquired with a scan rate of $100 \mathrm{~nm} / \mathrm{min}$. The morphology of the PAs was investigated using TEM. Notes for sample preparation procedures can be found in SI.

\subsection{Constructing the ECM-like multicomponent $2 D$ and $3 D$ hydrogels}

All PAs were dissolved with PBS, and the $\mathrm{pH}$ was adjusted to 7.4 with $0.1 \% \mathrm{NH}_{4} \mathrm{OH}$ or $\mathrm{HCl}$ (hydrogen chloride). To improve PA solubility, ultrasonic treatment was applied at $37{ }^{\circ} \mathrm{C}$. To prepare the co-assembled peptide gels for the observation of cell adhesion, $40 \mu \mathrm{L}$ of Osteo-PA and RGDS-PA/Osteo-PA (2\%) was separately placed in 96-wells, then calcium chloride $\left(\mathrm{CaCl}_{2}\right)(100 \mathrm{mM}, 2.5 \mu \mathrm{L})$ was added to trigger the hydrogelation. The resulting hydrogels were then washed with PBS for several times to remove excess calcium ions after $2 \mathrm{~h}$ incubation.

HA-Tyr/RGDS-PA/Osteo-PA/Angio-PA multicomponent structures were prepared as follow: $40 \mu \mathrm{L}$ HA-Tyr - previously synthesized and fully characterized [35]- (including 3U/mL HRP enzyme) placed in 96-wells. Osteo-PA and RGDS-PA (2\%), which had negative electrical charge like HA-Tyr, were injected into HA-Tyr at $5 \mu \mathrm{L}$ each, and followed by Angio-PA addition $(1 \%, 5 \mu \mathrm{L})$ which had positive charge. Note the Angio-PA molecules due to their large molecular weight and excessive hydrophobic amino acid residues cannot easily be dissolved when their concentration is higher than $1 \%$, therefore higher concentrations were not used. Addition of positively charged PA commenced the co-assembly reaction both with RGDS-PA, Osteo-PA and HA-Tyr. Lastly, a mixture of $\mathrm{CaCl}_{2}(100 \mathrm{mM}, 2.5 \mu \mathrm{L})$ and hydrogen peroxide $\left(\mathrm{H}_{2} \mathrm{O}_{2}\right)(1 \mathrm{mM}, 5 \mu \mathrm{L})$ was added that triggered an instant gelation via oxidation reaction where $\mathrm{H}_{2} \mathrm{O}_{2}$ and horseradish peroxidase (HRP) enzyme took place, in addition to the self-assembly mechanism. The obtained gels were $6.6 \mathrm{~mm}$ in diameter and $2 \mathrm{~mm}$ in height, resulting in a volume of approximately $68 \mathrm{~mm}^{3}$ (macro images can be seen in Figure S3). This system with gelling time of less than $1 \mathrm{~min}$ is thought to be suitable for 3D cell culture applications owing to its fast gelation property. The hydrogels were then washed with PBS for several times to remove excess $\mathrm{CaCl}_{2}$ and $\mathrm{H}_{2} \mathrm{O}_{2}$. HA-Tyr/RGDS-PA/Osteo-PA and HA-Tyr/RGDSPA/Angio-PA were prepared following the same way with excluding the PA not to be tested.

For 3D culture, same procedure with a slight change was applied. The cells (in $5 \mu \mathrm{L}$ medium) in this case were mixed with HA-Tyr in wells before PA additions, instead of seeding on top after gelation, to ensure a good homogeneity. PAs were injected in the aforementioned order, and the cross-linking reaction was set by adding $\mathrm{CaCl}_{2}$ and $\mathrm{H}_{2} \mathrm{O}_{2}$ subsequently. Since HA-Tyr works through a very fast gelation mechanism varying between $10 \mathrm{~s}$ and 1 min depending on 
1 the concentration, that is one of the great advantages of use of HA-Tyr for 3D culture, the gels

could be washed immediately after cross-linking that prevented cell damage.

\subsection{Characterization of ECM-like multicomponent hydrogels with Scanning Electron Microscope (SEM), rheometer, swelling and degradation tests}

Hydrogel microstructures with and without cells were characterized using SEM. Procedures for sample processing are provided in the SI.

The mechanical properties of HA-Tyr hydrogels were examined with oscillatory rheology instrument (Rheo DHR3, TA Instruments, USA). 1, 3, and 6\% HA-Tyr hydrogels were prepared, aged for $2 \mathrm{~h}$, and frequency sweep spectra were obtained. The stiffnesses of the gels were calculated using the mean $G^{\prime}$ value of the spectra.

For swelling potential of the hydrogels, three replicas of each dried hydrogel were swollen in PBS at room temperature for $24 \mathrm{~h}$ to achieve equilibrium swelling. After $24 \mathrm{~h}$, the hydrogels were removed from the solution and weighed after excessive solution on the surface was absorbed. The degree of swelling of hydrogels was measured with calculating the mass alteration using the following formula:

Degree of swelling $\left(\mathrm{Qm}_{\mathrm{m}}\right)=\left[\left(\mathrm{M}_{\mathrm{wet}}-\mathrm{M}_{\mathrm{dry}}\right) / \mathrm{M}_{\mathrm{dry}}\right] \times 100$

For the determination of degradation ability of the hydrogels, that is an important parameter for in vivo applicability of biomaterials for regenerative medicine purpose, the hydrogels $(\mathrm{n}=3)$ incubated in PBS at $37{ }^{\circ} \mathrm{C}$ overnight were weighed. Thereafter, hyaluronidase enzyme (200 U $\mathrm{mL}^{-1}$ ) was added in each well where the gels situated. After $12 \mathrm{~h}$ incubation in an incubator, the gels were weighed and mass losses (degradation) were calculated using the following formula:

Degradation \% $=\left[\left(M_{\text {initial }}-M_{\text {final }}\right) / M_{\text {initial }}\right] \times 100$

\subsection{Cell culture study}

The multicomponent hydrogels were treated with UV light in biosafety hood for $30 \mathrm{~min}$ for the sterilization before cell seeding. Human Adipose-Derived Mesenchymal Stem Cells (hAMSCs) purchased from Thermo Fisher (UK) were cultured in MesenPro medium (Thermo Fisher, UK) supplemented with antibiotic-antimycotic (1\%) and L-glutamine (200 mM, 1\%) under standard conditions $\left(5 \% \mathrm{CO}_{2}, 95 \%\right.$ humidity, and $\left.37^{\circ} \mathrm{C}\right)$, while Human Umbilical Vein Endothelial Cells (HUVECs, C-12203 Promocell, Germany) were cultured in M200 endothelial growth medium (Thermo Fisher, UK). Passages 3-6 were used for all the experiments. When the cells reached to $85-90 \%$ confluences, they were sub-cultured using trypsin-EDTA $(0.25 \%)$ and resuspended in culture medium. Depending on the application, varying cell densities were used including $5 \mathrm{~K}$ hAMSCs/gel in cell proliferation; $10 \mathrm{~K}$ hAMSCs/gel in osteogenic differentiation; and 5K, $10 \mathrm{~K}$, and $20 \mathrm{~K}$ hAMSCs/gel in the co-culture study. Also, 40K HUVECs/gel in the 2D/3D angiogenesis and $10 \mathrm{~K}, 20 \mathrm{~K}$, and $40 \mathrm{~K}$ HUVECs/gel in the co-culture study. The cells were gently seeded in growth media (GM) consisting of Dulbecco's Modified Eagle's Medium 
(DMEM), with Fetal Bovin Serum (FBS, 10\%), L-glutamine (1\%), and antibiotic-antimycotic solution (1\%) for hAMSCs and in M200 for HUVECs onto UV sterilized gels, and were incubated for $30 \mathrm{~min}$ at $37^{\circ} \mathrm{C}$ to allow the cells to settle onto the surface of the materials. Osteoinductive medium $(\mathrm{OIM})$ consisting of dexamethasone $(0.1 \mu \mathrm{M})$, sodium $\beta$-glycerophosphate $(10 \mathrm{mM})$, and ascorbic acid-2-phosphate $(0.05 \mathrm{mM})$ in basal medium (Lonza, Switzerland) was used for positive control experiments in osteogenic differentiation study.

\subsection{Calcein staining and cell proliferation study}

The suitability of HA-Tyr and HA-Tyr/RGDS-PA/Osteo-PA/Angio-PA co-assembled multicomponent hydrogel for cell culture applications and the effects of some parameters such as stiffness and PA concentrations on cell viability, adhesion, spreading, and qualitative cell proliferation were examined with calcein staining. After certain time points, media were removed from each 96-well, the hydrogels were washed with PBS, and calcein (stains live cells green) (Molecular Probes, Thermo Fisher, UK) solution $(4 \mu \mathrm{M})$ was added in each well. After 10 min incubation in the dark at $37^{\circ} \mathrm{C}$ and $5 \% \mathrm{CO}_{2}$, the cells were observed under SP2 Laser Scanning Confocal Microscope (LSCM) (Leica, Germany) using a $488 \mathrm{~nm}$ excitation wavelength. Three replicates of each gel were studied.

Proliferation of cells was measured with adenosine triphosphate (ATP) quantification by using Cell Titer-Glo (Promega, USA), which is a luminescent-based proliferation assay and is particularly suitable for 3D culture. After definite time points, the media were discarded and $100 \mu \mathrm{L}$ of reagent was added in each well. After $30 \mathrm{~min}$ incubation at room temperature, the luminescent signals were measured (485 nm excitation $/ 520 \mathrm{~nm}$ emission) with a FLUOstarOtpima FL luminescence microplate reader (BMG Labtech, Germany). Measurements for all types of gels namely HA-Tyr (2D and 3D), HA-Tyr with low PA concentrations (HA-Tyr/RGDS-PA(0.2\%)/Osteo-PA(0.2\%)/Angio-PA(0.1\%)) (2D) and high PA concentrations (HA-Tyr/RGDS-PA(2\%)/Osteo-PA(2\%)/Angio-PA(1\%)) (2D and 3D) were repeated three times.

\subsection{Angiogenesis assay}

In vitro angiogenic potential of 2D and 3D HA-Tyr/RGDS-PA/Angio-PA hydrogels were investigated by observing the branching and vascular tubule formation capability of cells. The prepared 2D hydrogels were UV-sterilized, and HUVECs were seeded in 40K cells/gel density on these gels as well as on Matrigel, which was studied as positive control. The aforementioned $3 \mathrm{D}$ gel preparation protocol was precisely applied for 3D angiogenesis assay. The cells in experimental groups were cultured in M200 endothelial cell growth medium, while VEGF (50 ng $\mathrm{mL}^{-1}$ ) was included to the medium for positive control experiments. The cells were stained with calcein after 3-days incubation for 2D and after 5-days incubation for 3D gels, and observed under SP2 LSCM (Leica, Germany). Confocal imaging for in vitro angiogenesis was studied with three replicates of gels.

\subsection{Pre-stained co-culture study}


1 For co-culture study, hAMSCs and HUVECs were pre-stained before seeding by incubating the cells with Dil or Dio ( $5 \mu \mathrm{L}$ in $1 \mathrm{~mL}$ cell suspension without FBS) for $10 \mathrm{~min}$. The excess dye was removed with centrifugation, and the cells were washed with resuspension/centrifugation for additional 2 times. Finally, the cells were seeded on (2D) or encapsulated in (3D) the gels $(n=3)$ in desired cell density.

\subsection{Immunofluorescence microscopy for osteogenic and angiogenic differentiation}

Immunofluorescence staining for osteopontin (Osn), alkaline phosphatase (ALP), and runtrelated transcription factor 2 (Runx2) antibodies for osteogenic differentiation in addition to CD31 and $\alpha$-smooth muscle actin ( $\alpha$ SMA) for angiogenic differentiation was carried out as described in SI.

\subsection{Gene expression study for angiogenic, osteogenic, and co-culture differentiation}

Osteogenic differentiation and angiogenesis were further investigated at the molecular level. Osteogenic and angiogenic gene expressions for Runx2 (osteogenic), CD31 and VEGF (angiogenesis) were examined after 14 days of culture for osteogenesis and 5 days of culture for angiogenesis. For co-culture differentiation, alteration in gene expression was investigated after a 14 days of co-culture. RNA isolation, cDNA synthesis, and reverse transcriptionquantitative polymerase chain reaction (RT-qPCR) protocols were provided in SI. Quantification of gene expressions of Runx2 (for ostegenic differentiation and co-culture study) as well as CD31 and VEGF (for angiogenesis and co-culture study) in 2D and 3D conditions were repeated three times.

\subsection{Statistical analysis}

All Live/Dead assays and proliferation tests were conducted three times. Statistical analysis was performed using Origin Pro 8. Statistical differences in gene expression results were determined using a two-way analysis of variance (ANOVA).

\section{Results}

\subsection{Synthesis and characterization of PAs and self-assembly}

Angio-PA, RGDS-PA, and Osteo-PA were synthesized by solid-phase peptide synthesis and purified by HPLC-MS as previously reported [36] and described in the SI. Zeta potentials of the synthesized PAs were measured to be Osteo-PA $(\zeta=-17.1 \mathrm{mV}), \operatorname{RGDS-PA}(\zeta=-56.0 \mathrm{mV})$, and Angio-PA $(\zeta=+24.0 \mathrm{mV})$ (Table S1). PA ( $0.1 \%$ wt.) self-assembly was characterized by CD spectroscopy. The CD spectra of Angio-PA, RGDS-PA, and Osteo-PA exhibited Compton effects with negative and positive bands at 205 and $220 \mathrm{~nm}$, respectively. These bands correspond to a $\beta$-sheet secondary structure (Figure 1c) [37]. TEM additionally was used to confirm the capacity of the individual PAs and their combinations to self-assemble into the classical high-aspect-ratio PA nanofibrous structure [12, 38] (Figure 1d). 


\subsection{Multicomponent hydrogel formation and characterization}

Stable hydrogels comprising all four HA-Tyr, Angio-PA, RGDS-PA, and Osteo-PA components were formed by means of an oxidation reaction via interaction between $\mathrm{H}_{2} \mathrm{O}_{2}$ and HRP enzyme in addition to the self-assembling mechanism (Figure S3). There was no significant difference in cell proliferation when $\mathrm{H}_{2} \mathrm{O}_{2}$ concentration was kept between 0.68$1.10 \mathrm{mM}$ [39]. Therefore, we used a fixed concentration of $\mathrm{H}_{2} \mathrm{O}_{2}(1 \mathrm{mM})$ and changed the concentrations of other components namely HA-Tyr and PAs in order to investigate cell behaviour. The hydrogels of HA-Tyr alone were optically transparent while those made from the individual PAs were translucent (Figure S3). Upon closer inspection via SEM, it was observed that HA-Tyr displayed a structure with a high density of micropores (Figure 1e,i) while the pure PA-based RGDS-PA/Osteo-PA/Angio-PA hydrogels exhibited the classical nanofibrous architecture of PA materials [12] (Figure 1e,iv). In contrast, the HA-Tyr/RGDSPA/Osteo-PA/Angio-PA hydrogel exhibited a combined microporous (Figure 1e,ii) and nanoporous (Figure 1e,iii) architecture.

Given the possibility to tune the hydrogels by modifying the amount of HA-Tyr used, we first conducted rheological measurements on HA-Tyr using concentrations of 1,3 , or $6 \%$. It should be noted that we could not test upper concentrations of HA-Tyr as it was not possible to pipette/transfer due to high viscosity. The viscosity of HA-Tyr $(3.5 \% \mathrm{w} / \mathrm{v})$ was measured to be $3.6 \mathrm{kPa} . \mathrm{s}$ in a previous report [40]. As expected, upon cross-linking with $1 \mathrm{mM} \mathrm{H}_{2} \mathrm{O}_{2}, 1,3$, and $6 \%$ hydrogels reached a G' of $0.65,1.8$, and $3.2 \mathrm{kPa}$, respectively (Figure S4). These values were then exploited for the calculation of elastic modulus, E, as described in the literature [41], which were found to be $1.95,5.40$, and $9.60 \mathrm{kPa}$ for 1,3 , and 6\% HA-Tyr, respectively. Thereafter, using the $6 \%$ HA-Tyr and $2 \%$ PA concentration, swelling and degradability tests were conducted on the multicomponent HA-Tyr/RGDS-PA/Osteo-PA/Angio-PA hydrogels by calculating changes in mass after either swelling the gels in PBS for $24 \mathrm{~h}$ or treating the gels with hyaluronidase enzyme for $12 \mathrm{~h}$. The results demonstrate that the multicomponent HATyr/RGDS-PA/Osteo-PA/Angio-PA hydrogels were less prone to swelling and enzymatic degradation compared to HA-Tyr (Figure S5 and 6). Swelling capacity decreased from $24.7 \%$ to $17.3 \%$ and degradation decreased from $20.4 \%$ to $2.5 \%$ on the multicomponent HATyr/RGDS-PA/Osteo-PA/Angio-PA compared to HA-Tyr.

\subsection{Cell adhesion and proliferation depend on hydrogel stiffness and composition}

To assess the potential applicability of the material as a cell culture scaffold, we conducted calcein staining in order to examine the cell viability, adhesion, and spreading by growing cells on (2D) and within (3D) the hydrogels. In addition, a luminescent-based proliferation assay (Cell Titer-Glo, Promega, USA) based on the quantification of adenosine triphosphate (ATP) was conducted to investigate cell proliferation. These experiments were conducted using different concentrations of PAs and HA-Tyr within the multicomponent hydrogels.

Although HA is a component of the ECM, it is well known that its distinctive hydrophilicity prevents cell adhesion (Figure S7) and consequently requires modification to support cell growth. To test the capacity of our hydrogels to enable cell growth, MSCs were cultured on preformed hydrogels for 7 days and stained with calcein to qualitatively assess viability as well 
as to observe cell adhesion and spreading under confocal microscope $[42,43]$. Based on the physical properties experiments, the concentration of the components was kept at $6 \% \mathbf{H A}-\mathbf{T y r}$ and 2\% RGDS-PA, 2\% Osteo-PA, and 1\% Angio-PA. As expected, MSCs growing on HATyr exhibited a spherical (non-spread) morphology and tended to aggregate (Figure S8), as has been previously reported [44]. In contrast, HA-Tyr/RGDS-PA/Osteo-PA/Angio-PA hydrogels presented many more cells, which exhibited spread morphology, likely due to the presence of RGDS-PA (Figure 2a,i). This hypothesis was confirmed by repeating the experiment on Osteo-PA and RGDS-PA/Osteo-PA, which also resulted in many more spread cells present on RGDS-PA/Osteo-PA (Figure 2a,ii). In order to reveal the effect of PA concentration on cell adhesion and spreading, the experiments were repeated using PA concentrations of 1:2 (1\% RGDS-PA, 1\% Osteo-PA, and 0.5\% Angio-PA) and 1:10 (0.2\% RGDS-PA, $0.2 \%$ Osteo-PA, and $0.1 \%$ Angio-PA). The results demonstrate that cell adhesion and spreading increased with ascending PA concentration (Figure 2a,iii). SEM observations further verified the better cell adhesion and spreading on the multicomponent HA-Tyr/RGDSPA/Osteo-PA/Angio-PA compared to HA-Tyr (Figure S9).

To further assess the potential of the gels to serve as $2 \mathrm{D}$ cell culture substrates, we tested the proliferation of MSCs growing on gels comprising 6\% HA-Tyr and varying PA concentrations. Cell number increased from day 1 to 7 on the multicomponent HA-Tyr/RGDS-PA/OsteoPA/Angio-PA hydrogels (Figure 2b). Interestingly, while HA-Tyr exhibited less number of cells at day 7, cells on these hydrogels exhibited similar levels of cell proliferation (Figure 2b) to those of cells growing on HA-Tyr/RGDS-PA/Osteo-PA/Angio-PA. Similar to the cell adhesion results, cell proliferation also decreased with decreasing PA concentration (Figure $2 \mathrm{~b}$ ), thus $2 \% \mathrm{PA}$ concentration was used in $3 \mathrm{D}$ cell culture experiments.

Based on the 2D experiments, hydrogels were formed using 2\% PA concentration and varying concentrations of HA-Tyr (1, 3, or 6\%) in order to assess the effect of hydrogel stiffness, a key parameter in 3D cell culture [45]. Hydrogels were self-assembled in the presence of cells so as to embed the cells at the start of the culture. Confocal imaging revealed that cells were present and viable in both HA-Tyr and HA-Tyr/RGDS-PA/Osteo-PA/Angio-PA with higher number of cells on the multicomponent hydrogel (Figure 3a). However, in contrast to cells growing within HA-Tyr, cells within HA-Tyr/RGDS-PA/Osteo-PA/Angio-PA after 7 days of culture were spread, likely as a result of the presence of RGDS-PA. While cell spreading within 3D gels is not common, previous studies have reported MSC spreading within a variety of hydrogels [46-49]. Interestingly, both cell number and cell spreading in 3D were higher in multicomponent hydrogels exhibiting higher concentration of HA-Tyr (Figure S10). These observations were supported by the cell proliferation assay as it revealed both higher proliferation rates within HA-Tyr/RGDS-PA/Osteo-PA/Angio-PA compared to HA-Tyr (Figure $3 \mathrm{~b}$ ) as well as higher proliferation rates in multicomponent hydrogels comprising higher HA-Tyr concentrations (Figure S11). Unless otherwise specified, 6\% HA-Tyr and 2\% PA concentrations were used in downstream experiments.

\subsection{Gel stimulates vascular tubule formation in vitro in $2 D$ and $3 D$}


1 To test the angiogenic potential of the hydrogels, HUVECs were seeded on the hydrogels, cultured for 3 days in endothelial cell culture medium, and assessed for tubule formation. In this case, we tested the multicomponent hydrogels HA-Tyr/RGDS-PA/Angio-PA (using 6\% HA-Tyr, 2\% RGDS-PA, and 0.5\% Angio-PA considering the highest solubility (1\%) of AngioPA) in order to better assess the specific angio-inductive stimuli, without the presence of the osteogenic one. First, as expected, no angiogenesis or branching was observed on HA-Tyr (Figure 4a.i). However, upon incorporation of RGDS-PA in the system (HA-Tyr/RGDS-PA) (negative control), cells adhered and spread (Figure 4a.ii) and on further incorporation of Angio-PA (HA-Tyr/RGDS-PA/Angio-PA), cells not only adhered and spread but also exhibited clear branching (Figure 4a.iii), suggesting a strong effect from the Angio-PA. To confirm this hypothesis, we repeated the experiments doubling the concentration of the PAs (1\%) and indeed cells were able to form large vascular lumens (Figure 4a.iv). Remarkably, this level of angiogenesis was similar to that of cells cultured on the positive control Matrigel in the presence of VEGF (50 $\mathrm{ng} \mathrm{mL}^{-1}$ ) (Figure 4a.v). In contrast, cells growing on tissue culture plastic (TCP, negative control) exhibited a solely adhered monolayer without any signs of angiogenesis (Figure 4a.vi).

To further assess the angiogenic potential of HA-Tyr/RGDS-PA/Angio-PA, phenotypic characterization of angiogenic organization and tubule formation were investigated on HUVECs cultured for 5 days. Immunofluorescence staining of the two angiogenic markers CD31 and $\alpha$ SMA was performed on cells growing on HA-Tyr/RGDS-PA/Angio-PA and TCP (negative control). Here, 6\% HA-Tyr and 2\% PAs were used as they were previously identified as the optimal concentrations for cell adhesion and proliferation in $2 \mathrm{D}$ and $3 \mathrm{D}$. The results indicate that cells on HA-Tyr/RGDS-PA/Angio-PA co-expressed CD31 and $\alpha$ SMA, but with weaker $\alpha$ SMA compared to CD31, which indicates cells organizing and forming functional, yet not fully matured, tubules (Figure 4b). In contrast, cells on TCP retained their monolayer property and did not express $\alpha$ SMA, indicating a non-induced phase. In addition, tubule formation was also assessed on cells growing on HA-Tyr/RGDS-PA/Angio-PA and HATyr/RGDS-PA (negative control) by quantifying the gene expression of CD31 and VEGF. The results revealed an increase in these markers for cells growing on HA-Tyr/RGDS-PA/AngioPA compared to cells on HA-Tyr/RGDS-PA (Figure 4c), which supports the immunofluorescence results (Figure $4 \mathrm{~b}$ ) and confirms the bioactivity of the developed SVVYGLR epitope presenting nanofibres.

In order to examine the angio-inductive properties of HA-Tyr/RGDS-PA/Angio-PA (6\% HATyr, $2 \%$ RGDS-PA, and 1\% PAs) in 3D, HUVECs were encapsulated in the hydrogels, cultured for 5 days in endothelial cell culture medium, and stained with calcein to assess cell morphology. Confocal imaging revealed that cells encapsulated in HA-Tyr/RGDS-PA/AngioPA formed branched 3D structures (Figure 4d). On the contrary, cells growing within HA-Tyr (negative control) remained viable but formed aggregates instead of branching (Figure 4d, S2D image).

\subsection{Gel induces hAMSCs towards an osteogenic lineage}


1 Another goal of this work was to design a hydrogel that, in addition to promote angiogenesis,

is able to direct cells towards an osteoblastic lineage. To this end, phenotypic changes of MSCs growing on the hydrogels in growth medium (GM) were investigated with immunofluorescence microscopy for the osteogenic markers Osn, ALP, and Runx2. In this case, we tested HATyr/RGDS-PA/Osteo-PA (using 6\% HA-Tyr and 2\% PAs) in order to better assess the specific osteo-inductive stimuli. We observed that cells cultured on HA-Tyr/RGDS-PA/Osteo-PA hydrogels began to express osteogenic markers after day 14 of culture (Figure 5a,i-iii). The expression of these markers appeared to be higher than that of cells growing on HATyr/RGDS-PA (negative control) (Figure 5a,iv-vi) in GM but lower than cells on the positive control Matrigel in OIM (Figure 5a,vii).

To corroborate the level of osteogenic differentiation observed on and within our hydrogels, RT-qPCR was used to study the early osteogenic marker Runx2 gene, which was highly expressed in the immunofluorescence study. The experiments were conducted on the same groups as detailed above and both in $2 \mathrm{D}$ and $3 \mathrm{D}$. The results in $2 \mathrm{D}$ demonstrate that cells growing on HA-Tyr/RGDS-PA/Osteo-PA (in GM) exhibited a seven-fold increase in Runx2 expression compared to HA-Tyr/RGDS-PA (in GM) (negative control) (Figure 5b). However, the level of osteogenic differentiation was lower than cells growing on the same HATyr/RGDS-PA/Osteo-PA hydrogels as well as in Matrigel but in OIM (Figure 5b). In 2D, Runx2 expression of cells on HA-Tyr/RGDS-PA/Osteo-PA (in OIM) was lower than that those on Matrigel in OIM. However, in 3D, Runx2 expression of cells growing within HATyr/RGDS-PA/Osteo-PA (in OIM) was higher than cells growing in Matrigel (in OIM) (Figure 5c).

\subsection{Gel as a bone-vascular cell co-culture environment in $2 D$ and $3 D$}

The potential of the hydrogels to enable hybrid bone-vascular cell co-cultures was investigated by co-culturing pre-stained HUVECs (red) and hAMSCs (green). Here, we harnessed the simplicity of multicomponent self-assembly to fabricate angio-inductive and osteo-inductive hydrogels. Cells were cultured at a ratio of 10K:10K (hAMSCs:HUVECs) on the surface of (2D) and within (3D) HA-Tyr/RGDS-PA/Osteo-PA/Angio-PA.

In 2D, at day 5, cells formed random cell aggregates on the HA-Tyr (Figure 6a.i). In contrast, cells growing on the HA-Tyr/RGDS-PA/Osteo-PA/Angio-PA were homogenously distributed and exhibited a more adhered and spread morphology (Figure 6a.ii). However, no HUVEC sprouting nor branching were observed (Figure 6a.ii). When the cells were co-cultured at a ratio of $5 \mathrm{~K}: 20 \mathrm{~K}$, enhanced angiogenesis was observed evidenced by emergence of HUVEC sprouting (arrow), branching (arrowhead), and lumen formation (star), while a small number of hAMSCs adhered (Figure 6a.iii). However, when doubling the cell number (10K:40K), large vascular lumens were observed (in red) encircling a much larger number of differentiating hAMSCs (in green) (Figure 6a.iv). This result further demonstrates that HA-Tyr/RGDSPA/Osteo-PA/Angio-PA is able to promote a vascularized bone-like organization. Interestingly, this phenomenon was also observed when cells were co-cultured at a $10 \mathrm{~K}: 40 \mathrm{~K}$ ratio in 3D (Figure 6b). In this case, HUVECs and hAMSCs were encapsulated in the HATyr/RGDS-PA/Osteo-PA/Angio-PA. After 3 days of co-culture, cells growing within HA- 
1 Tyr/RGDS-PA/Osteo-PA/Angio-PA were well-connected (arrow), formed fused cell clumps (arrowhead), and constructed tubule-like extensions (star).

To further examine the potential of the multicomponent system to induce the desired bone-like construct from differentiating hAMSCs/HUVECs, gene expression analyses of Runx2, CD31, and VEGF were performed at day 14. These osteogenic (Runx2) and angiogenic (CD31 and VEGF) markers were investigated after hAMSCs and HUVECs (10K:40K) were co-cultured either on (2D) or within (3D) the different materials, as well as in different conditions including $\mathrm{GM}+\mathrm{M} 200$ (experimental group) and OIM+M200 (positive control for osteoinduction). The cells that were seeded on or within HA-Tyr/RGDS-PA/Osteo-PA/Angio-PA and cultured in GM+M200 expressed all markers (Figure 6c-h). Compared to TCP (negative control), HATyr/RGDS-PA/Osteo-PA/Angio-PA in 2D showed higher Runx2 expression in co-culture growth medium (GM+M200) (Figure 6c). However, this expression was lower than that on the same material (HA-Tyr/RGDS-PA/Osteo-PA/Angio-PA) but in co-culture differentiation medium (OIM+M200) (Figure 6c). Furthermore, expression of Runx2 was found to be highest in cells growing on and within Matrigel (Figure 6c,f). On the other hand, higher expression of angiogenic markers was observed in cells growing on (Figure 6d,e) or within (Figure 6g,h) HATyr/RGDS-PA/Osteo-PA/Angio-PA compared to Matrigel (in OIM+M200) and HATyr/RGDS-PA (in GM+M200) (negative control) (Figure 6d,e).

In 3D, we compared the HA-Tyr/RGDS-PA/Osteo-PA/Angio-PA (in GM+M200) with HATyr (negative control) (in GM+M200) and Matrigel (OIM+M200). In this case, higher levels of angiogenic markers were observed in HA-Tyr/RGDS-PA/Osteo-PA/Angio-PA compared to HA-Tyr and Matrigel (Figure 6g,h).

\section{Discussion}

In this study, we designed different types of self-assembling multicomponent hydrogels to support cell proliferation, adhesion, osteogenic differentiation, vascular organization, and hybrid bone-vascular co-culture formation. These multifunctional gels not only offer an opportunity to recreate some of the molecular complexity found in the native ECM but also the potential to enhance vascularization in tissue engineering and regenerative medicine applications. We hypothesized that co-assembly between the different PAs would be primarily driven by electrostatic interactions between the oppositely charged Osteo-PA $(\zeta=-17.1 \mathrm{mV})$, RGDS-PA $(\zeta=-56.0 \mathrm{mV})$, and Angio-PA $(\zeta=+24.0 \mathrm{mV})$ (Table S1). Similar co-assembly between oppositely charged PAs has been previously demonstrated [50]. In our case, both CD spectra and TEM observations confirmed formation of the classical PA nanofiber architecture (Figure 1c,d).

To achieve homogenous integration of the different components, hydrogels were prepared by first mixing the negatively charged components HA-Tyr (6\%), RGDS-PA (2\%), and OsteoPA (2\%) and then injecting the positively charged Angio-PA (1\%) into the mixture to trigger co-assembly and initial gel formation. Then, complete gelation was triggered by immediately injecting a mixed solution of $\mathrm{CaCl}_{2}(100 \mathrm{mM})$ and $\mathrm{H}_{2} \mathrm{O}_{2}(1 \mathrm{mM})$ in order to obtain robust gels 
1 by means of ionic interactions and covalent crosslinking. Previous studies have reported that 2 HA-Tyr hydrogels require $\mathrm{H}_{2} \mathrm{O}_{2}$ concentrations of $0.65 \mathrm{mM}$ or higher to maintain the 3 viscoelastic properties [39]. This is an important parameter to control as MSC morphology has 4 been reported to change depending on the concentration of $\mathrm{H}_{2} \mathrm{O}_{2}$ in HA-Tyr gels [39]. 5 Consequently, we kept constant the concentration of $\mathrm{H}_{2} \mathrm{O}_{2}$ at $1 \mathrm{mM}$ and systematically modified 6 PA and HA-Tyr concentrations in order to investigate their effect on cell adhesion, 7 8 9 10 proliferation, and viability. Through this approach, various multicomponent self-assembling gels using 1, 3, and 6\% HA-Tyr and 0.2-2\% PAs were obtained, exhibiting storage moduli between $0.65-3.20 \mathrm{kPa}$. It is important to mention that relatively soft gels [44, 51], including PA nanofiber-based gels [25], have been found to support the growth of cells from hard tissues including stimulation of tissue regeneration in vivo [52]. This is likely a result of the soft scaffolds enabling cells to sense mechanical changes, remodel, and hereby help tissue regeneration.

An ideal ECM-mimicking material should facilitate cell infiltration and growth. Our results demonstrate that HA-Tyr/RGDS-PA/Osteo-PA/Angio-PA promoted cell adhesion, spreading, and proliferation compared to HA-Tyr (Figure 2). The structural properties of the hydrogel play a key role in this context [25, 51]. The multicomponent HA-Tyr/RGDS-PA/OsteoPA/Angio-PA hydrogel exhibited lower swelling capacity (from $24.7 \%$ to $17.3 \%$ ) and degradation (from $20.4 \%$ to $2.5 \%$ ) compared to HA-Tyr hydrogels. We speculate that this enhanced stability results from interactions between the PA nanofibers and the HA-Tyr backbone and is likely playing role in the difference in cell behaviors between the two hydrogels. In addition, the results also suggest that the presence of the bioactive PAs is also essential as cell proliferation decreased on multicomponent hydrogels with decreasing concentrations of Pas. Interestingly, while cells on HA-Tyr exhibited decreased adhesion and spreading, they maintained a high proliferative state, which may arise from cell-to-cell and cellmatrix interactions as has been previously reported [53].

The 3D structure of the native ECM plays a pivotal role on stem cell maintenance and differentiation [46]. To assess the capacity of the hydrogels to embed cells and support their growth in 3D, calcein staining and MSC proliferation within the gels was quantified. In both $2 \mathrm{D}$ and 3D, RGDS was determined as the key element for cell adhesion and spreading. On the other hand, both cell number and cell spreading in 3D were higher in multicomponent hydrogels exhibiting higher concentration of HA-Tyr (Figure 3). These results are in alignment with previous studies that have reported higher metabolic activities of cells growing within stiffer hydrogels [46] and in the presence of PAs [54], further evidencing potential benefits of our multicomponent approach. These results demonstrate that hydrogels comprising 6\% HA-Tyr and 2\% PA concentrations elicit optimum levels of cell adhesion, spreading, and proliferation both in 2D and 3D (Figure 2 and 3).

To test the gels' angio-inductive and osteo-inductive potential, we compared HA-Tyr/RGDSPA/Angio-PA and HA-Tyr/RGDS-PA/Osteo-PA with the relevant control gels. Remarkably, the level of angiogenic organization triggered by HA-Tyr/RGDS-PA/Angio-PA in 2D and 3D was similar to that of cells growing within the positive control Matrigel in the presence of VEGF (Figure 4). In addition, HA-Tyr/RGDS-PA/Osteo-PA induced hAMSCs into osteoblastic 
1 lineage in 2D and 3D (Figure 5). These findings are in alignment with previous efforts reporting autonomous osteogenic differentiation of MSCs [25,55,56]. Furthermore, osteogenic gene (Runx2) expression in cells growing on HA-Tyr/RGDS-PA/Osteo-PA was higher in 3D compared to 2D (Figure 5). This difference in Runx2 expression of cells growing in 2D and 3D might be attributed to advantages of 3D architecture provided by RGDS-PA and Osteo-PA. Similar observations asserting that cells express higher levels of osteogenic markers in 3D PA hydrogels has been reported [25].

The potential of the hydrogels to enable hybrid bone-vascular cell co-cultures was investigated by co-culturing pre-stained HUVECs (red) and hAMSCs (green) (Figure 6). Such co-cultures have been conducted through sophisticated techniques such as bioprinting [57] or lithography [58]. hAMSCs and HUVECs, when seeded on HA-Tyr/RGDS-PA/Osteo-PA/Angio-PA (2D) in $10 \mathrm{~K}: 40 \mathrm{~K}$ cell ratio, triggered a cellular organization consisting of large vascular lumens encircling the differentiating MSCs (Figure 6). Similar vascular sprouting and branching have been reported when co-culturing HUVECs with MSCs but only in the presence of VEGF [57]. On the other hand, when the cells were seeded in 3D, a bone-vascular micro-tissue with tubulelike extensions was observed (Figure 6). Previous studies have demonstrated the dependence of HUVECs on growth factors such as HepG2 [59] and VEGF [60] and the capacity of Matrigel to promote angiogenesis in these conditions. In contrast, here we demonstrate that the multicomponent HA-Tyr/RGDS-PA/Osteo-PA/Angio-PA provides a growth factor-free environment with the capacity to promote angiogenic differentiation.

Despite the promising results obtained in this study, further optimization of the material is required. First, while the immunofluorescence (Runx2, ALP, Osn) and gene expression (Runx) results demonstrate proof-of-concept of osteogenic and angiogenic induction, investigation of more markers would be required to fully charaterize the inductive capacity of the material. Also, bone is a complex tissue where multiple types of cells such as osteoblasts, osteocytes, and osteoclasts interact in a coordinated manner. Proper recreation of the bone environment should include all these cell types. On the other hand, PAs offer unique advantages as a result of their self-assembling nature including the formation of well-defined nanofibers, pores for diffusion and cell migration, and the display of multiple bioactive epitopes. However, these molecules can also elicit some toxic reactions when used at high concentrations or depending on their molecule sequence. For example, reducing alkyl tail length or increasing the $\beta$-sheet strength decrease cell toxicity [61]. Therefore, the multicomponent system could be optimized.

Lastly, as seen from our characterization of rheological properties, the materials are able to support cell growth. However, the use of this material for in vivo applications is limited given the requirement of many hard tissue applications for much robust mechanical properties. Furthermore, its use as a $3 \mathrm{D}$ printing bioink requires further optimization to enable faster setting properties. Nonetheless, we have demonstrated that the multicomponent HA-Tyr/RGDSPA/Osteo-PA/Angio-PA material offers a high level of tunable bioactivity that has the capacity to guide multiple cell populations to form an osteogenic environment. Furthermore, the results confirm the potential of the multicomponent approach to not only endow bioactivity but also enhance the structural integrity of the gels. While these characteristics are ideal to develop more 
3

5

complex in vitro models, the material may also be used as a component of in vivo therapeutic strategies that require enhanced bioactivity.

\section{Conclusion}

Bioactive environments that can recreate key properties of the native ECM and induce multiple cell types are essential for the kind of cell differentiation and organization properties required in effective in vitro models. We have developed a multifunctional hydrogel designed to promote osteogenesis and angiogenesis by recreating key structural and signaling elements of the native bone environment. The system takes advantage of both non-covalent and covalent interactions and enables the incorporation of specific bioactive epitopes and ECM components within a nanofibrous and microporous architecture. We have demonstrated how the multicomponent HA-Tyr/RGDS-PA/Osteo-PA/Angio-PA hydrogel can promote hAMSC adhesion and osteoblastic differentiation in addition to inducing HUVECs to grow into vascular tubules. By co-culturing these cells, we verify the capacity of the hydrogel to generate bone-like constructs in vitro. The results demonstrate the potential of these hydrogels to serve as complex in vitro environments as well as potential in vivo materials for bone tissue engineering.

\section{Acknowledgements}

This work was financially supported by the Scientific and Technological Research Council of Turkey (TUBITAK) with the 1059B191601219 numbered project, the ERC Starting Grant (STROFUNSCAFF), AO Research Fund of the AO Foundation project number AOCMF-1719M, and the UK Regenerative Medicine Platform (UKRMP2) Acellular Smart Materials.

\section{Declaration of Competing Interest}

The authors declare no conflict of interest.

\section{References}

[1] A.M. Rosales, K.S. Anseth, The design of reversible hydrogels to capture extracellular matrix dynamics, Nat. Rev. Mater. 1 (2016) 15012.

[2] M.J. Webber, E.A. Appel, E.W. Meijer, R. Langer, Supramolecular biomaterials, Nat. Mater. 15 (2016) 13.

[3] Y.E. Arslan, Y.F. Galata, T.S. Arslan, B. Derkus, Trans-differentiation of human adiposederived mesenchymal stem cells into cardiomyocyte-like cells on decellularized bovine myocardial extracellular matrix-based films, J. Mater. Sci: Mater. Med. 29(8) (2018) 127. 
[4] A.E. Jakus, A.L. Rutz, S.W. Jordan, A. Kannan, S.M. Mitchell, C. Yun, K.D. Koube, S.C. Yoo, H.E. Whiteley, C-P. Richter, R.D. Galiano, W.K. Hsu, S.R. Stock, E.L. Hsu, R.N. Shah, Hyperelastic "bone": A highly versatile, growth factor-free, osteoregenerative, scalable, and surgically friendly biomaterial, Sci. Transl. Med. 8(358) (2016) 358ra 127.

[5] A. Mata, Y. Geng, K.J. Henrikson, C. Aparicio, S.R. Stock, R.L. Satcher, S.I. Stupp, Bone regeneration mediated by biomimetic mineralization of a nanofiber matrix, Biomaterials 31(23) (2010) 6004 .

[6] L. Shi, F. Wang, W. Zhu, Z. Xu, S. Fuchs, J. Hilborn, L. Zhu, Q. Ma, Y. Wang, X. Weng, and D.A. Ossipov, Self-healing silk fibroin-based hydrogel for bone regeneration: Dynamic metal-ligand self-assembly approach, Adv. Funct. Mater. 27 (2017) 1700591.

[7] A.C. Mendes, K.H. Smith, E. Tejeda-Montes, E. Engel, R.L. Reis, H.S. Azevedo, A. Mata, Co-assembled and microfabricated bioactive membranes, Adv. Func. Mater. 23(4) (2013) 430.

[8] E. Tejeda-Montes, A. Klymov, M.R. Nejadnik, M. Alonso, J.C. Rodriguez-Cabello, X.F. Walboomers, A. Mata, Mineralization and bone regeneration using a bioactive elastin-like recombinamer membrane, Biomaterials 35(29) (2014) 8339.

[9] K. Zhang, Q. Feng, J. Xu, X. Xu, F. Tian, K.W.K. Yeung, and L. Bian, Self-Assembled Injectable Nanocomposite Hydrogels stabilized by bisphosphonate-magnesium (Mg2+) coordination regulates the differentiation of encapsulated stem cells via dual crosslinking, Adv. Funct. Mater. 27 (2017) 1701642.

[10] M. Dang, L. Saunders, X. Niu, Y. Fun, P.X. Ma, Biomimetic delivery of signals for bone tissue engineering, Bone Res. 6 (2018) 25.

[11] S. Zhang, Fabrication of novel biomaterials through molecular self-assembly, Nat. Biotechnol. 21 (2003) 1171.

[12] J.D. Hartgerink, E. Beniash, and S.I. Stupp. Peptide-amphiphile nanofibers: A versatile scaffold for the preparation of self-assembling materials, Proc. Natl. Acad. Sci. 99(8) (2002) 5133.

[13] M.A. Biesalski, A. Knaebel, R. Tu, M. Tirrell, Cell adhesion on a polymerized peptideamphiphile monolayer, Biomaterials 27(8) (2006) 1259.

[14] M. Mata, L. Hsu, R. Capito, C. Aparicio, C. Henrikson, and S.I. Stupp, Micropatterning of bioactive self-assembling gels, Soft Matter 5 (2009) 1228.

[15] D. Eren, G. Tansik, A.B. Tekinay, M.O. Guler. Mineralized Peptide Nanofiber Gels for Enhanced Osteogenic Differentiation, ChemNanoMat 4(8) (2018) 837. 
[16] S. Debnath, S. Roy, R.V. Ulijn, Peptide nanofibers with dynamic instability through nonequilibrium biocatalytic assembly, J. Am. Chem. Soc. 135(45) (2013) 16789.

[17] G.A. Hudalla, T. Sun, J.Z. Gasiorowski, H. Han, Y.F. Tian, A.S. Chong, and J.H. Collier, Gradated assembly of multiple proteins into supramolecular nanomaterials, Nat. Mater. 13 (2014) 829.

[18] B.O. Okesola, A.Mata, Multicomponent self-assembly as a tool to harness new properties from peptides and proteins in material design, Chem. Soc. Rev. 47(10) (2018) 3721.

[19] E.R. Draper, and D.J. Adams, How should multicomponent supramolecular gels be characterised?, Chem. Soc. Rev. 47 (2018) 3395.

[20] H. Cheng, Y.J. Cheng, S. Bhasin, J.Y. Zhu, X.D. Xu, R.X. Zhuo, X.Z. Zhang, Complementary hydrogen bonding interaction triggered co-assembly of an amphiphilic peptide and an anti-tumor drug, Chem. Commun. (Camb). 51(32) (2015) 6936.

[21] R.M. Capito, H.S. Azevedo, Y.S. Velichko, A. Mata, S.I. Stupp, Self-assembly of large and small molecules into hierarchically ordered sacs and membranes, Science 319 (2008) 1812.

[22] K.E. Inostroza-Brito, E. Collin, O. Siton-Mendelson, K.H. Smith, A. Monge-Marcet, D.S. Ferreira, R.P. Rodríguez, M. Alonso, J.C. Rodríguez-Cabello, R.L. Reis, F. Sagués, L. Botto, R. Bitton, H.S. Azevedo, A. Mata, Co-assembly, spatiotemporal control and morphogenesis of a hybrid protein-peptide system, Nat. Chem. 7(11) (2015) 897.

[23] R. Freeman, M. Han, Z. Álvarez, J.A. Lewis, J.R. Wester, N. Stephanopoulos, M.T. McClendon, C. Lynsky, J.M. Godbe, H. Sangji, E. Luijten, S.I. Stupp, Reversible selfassembly of superstructured networks, Science 362 (2018) 808.

[24] T. Jiang, T.A. Meyer, C. Modlin, X. Zuo, V.P. Conticello, Y. Ke, Structurally ordered nanowire formation from co-assembly of DNA origami and collagen-mimetic peptides, J. Am. Chem. Soc. 139(40) (2017) 14025.

[25] N. Mari-Buye, T. Luque, D. Navajas, and C.E. Semino, Development of a threedimensional bone-like construct in a soft self-assembling peptide matrix, Tissue Eng. A 19(78) (2013) 870 .

[26] S.Y. Yoo, M. Kobayashi, P.P. Lee, and S-W. Lee, Early osteogenic differentiation of mouse preosteoblasts induced by collagen-derived DGEA-peptide on nanofibrous phage tissue matrices, Biomacromolecules 12 (2011) 987.

[27] J.M. Anderson, J.B. Vines, J.L. Patterson, H. Chen, A. Javed, H-W. Jun, Osteogenic differentiation of human mesenchymal stem cells synergistically enhanced by biomimetic peptide amphiphiles combined with conditioned medium, Acta Biomater. 7 (2011) 675. 
[29] X. Wang, A. Horii, and S. Zhang, Designer functionalized self-assembling peptide endothelial cells, Soft Matter 4 (2008) 2388.

[30] A.N. Moore, T.L.L. Silva, N.C. Carrejo, C.A.O. Marmolejo, C. Li, J.D. Hartgerink, Nanofibrous peptide hydrogel elicits angiogenesis and neurogenesis without drugs, proteins, or cells, Biomaterials 161 (2018) 154.

[31] Y. Hamada, K. Nokihara, M. Okazaki, W. Fujitani, T. Matsumoto, M. Matsuo, Y. Umakoshi, J. Takahashi, and N. Matsuura, Angiogenic activity of osteopontin-derived peptide SVVYGLR, Biochem. Biophys. Res. Commun. 310 (2003) 153.

[32] K.M. Park, Y. Lee, J.Y. Son, J.W. Bae, and K.D. Park, In situ SVVYGLR peptide conjugation into injectable gelatin-poly(ethylene glycol)-tyramine hydrogel via enzymemediated reaction for enhancement of endothelial cell activity and neo-vascularization, Bioconj. Chem. 23 (2012) 2042.

[33] D. Park, Y. Kim, H. Kim, K. Kim, Y-S. Lee, J. Choe, J-H. Hahn, H. Lee, J. Jeon, C. Choi, Y-M. Kim, D. Jeoung, Hyaluronic acid promotes angiogenesis by inducing RHAMM-TGF $\beta$ receptor interaction via CD44-PKC 8 , Mol. Cells 33 (2012) 563.

[34] C. Loebel, S.E. Szczesny, B.D. Cosgrove, M. Alini, M. Zenobi-Wong, R.L. Mauck and D. Eglin, Cross-linking chemistry of tyramine-modified hyaluronan hydrogels alters mesenchymal stem cell early attachment and behavior, Biomacromolecules 18 (2017) 855.

[35] C. Loebel, T. Stauber, M. D'Este, M. Alini, M. Zenobi-Wong, D. Eglin, Fabrication of cellcompatible hyaluronan hydrogels with a wide range of biophysical properties through high tyramine functionalization, J. Mater. Chem. B, 5 (2017) 2355.

[36] A. Mata, L. Palmer, E. Tejeda-Montes, S.I. Stupp, Design of biomolecules for nanoengineered biomaterials for regenerative medicine, Methods Mol. Biol. 811 (2012) 39.

[37] N. Greenfield, G.D. Fasman, Computed circular dichroism spectra for the evaluation of protein conformation, Biochemistry 8 (1969) 4108.

[38] J.D. Hartgerink, E. Beniash, S.I. Stupp. Self-assembly and mineralization of peptideamphiphile nanofibers, Science 294 (2001) 1684. 

hyaluronan bioink through enzymatic-and visible light-crosslinking, Biofabrication. 25;10(4) (2018) 044104.

[40] D. Petta, D.W. Grijpma, M. Alini, D. Eglin, M. D’Este, Three-dimensional printing of a tyramine hyaluronan derivative with double gelation mechanism for independent tuning of shear thinning and postprinting curing, ACS Biomater. Sci. Eng., 4 (2018) 3088.

[41] L.N. Beenken-Rothkopf, L.S. Karfeld-Sulzer, N.E. Davis, R. Forster, A.E. Barron, and M.J. Fontaine, The incorporation of extracellular matrix proteins in protein polymer hydrogels to improve encapsulated beta-cell function, Ann. Clin. Lab. Sci. 43(2) (2013) 111-121.

[42] M. Maeda, M. Hirose, O. Ohgoshi, T. Kirita, In vitro mineralization by mesenchymal stem cells cultured on titanium scaffolds. J. Biochem. 141 (2007) 729.

[43] N. Kohli, K.T. Wright, R.L. Sammons, L. Jeys, M. Snow, W.E.B. Johnson, An in vitro comparison of the incorporation, growth, and chondrogenic potential of human bone marrow versus adipose tissue mesenchymal stem cells in clinically relevant cell scaffolds used for cartilage repair, Cartilage 6(4) (2015) 252.

[44] E. Jooybar, M.J. Abdekhodaie, M. Alvi, A. Mousavi, M. Karperien, P.T. Dijkstra, An injectable platelet lysate-hyaluronic acid hydrogel supports cellular activities and induces chondrogenesis of encapsulated mesenchymal stem cells, Acta Biomater. 83 (2019) 233.

[45] E. Shamir, A.J. Ewal, Three-dimensional organotypic culture: experimental models of mammalian biology and disease, Nat. Rev. Mol. Cell Biol. 15 (2014) 647.

[46] G. Brustain, T. Panciera, A. Gandin, A. Citron, S. Piccolo, Biomaterials and engineered microenvironments to control YAP/TAZ-dependent cell behaviour, Nat. Mater. 17 (2018) 1063.

[47] J. Lou, R. Stowers, S. Nam, Y. Xia, O. Chaudhuri, Stress relaxing hyaluronic acidcollagen hydrogels promote cell spreading, fiber remodeling, and focal adhesion formation in 3D cell culture, Biomaterials 154 (2018) 213.

[48] P.J. Yang, M.E. Levenston, and J.S. Temenoff, Modulation of mesenchymal stem cell shape in enzyme-sensitive hydrogels is decoupled from upregulation of fibroblast markers under cyclic tension, Tissue Eng. A 18 (2012) 2365.

[49] N.J. Hogrebe, J.W. Reinhardt, N.K. Tram, A.C. Debski, G. Agarwal, M.A. Reilly, K.J. Gooch, Independent control of matrix adhesiveness and stiffness within a 3D self-assembling peptide hydrogel, Acta Biomater. 70 (2018) 110.

[50] H.A. Behanna, J.J.J.M. Donners, A.C. Gordon, S.I. Stupp, Coassembly of amphiphiles with opposite peptide polarities into nanofibers, J. Am. Chem. Soc. 127(4) (2005) 1193. 

Substrate stress-relaxation regulates scaffold remodeling and bone formation in vivo, Adv. Healthcare Mater. 6 (2017) 1601185.

5

[52] B.O. Okesola, S. Ni, B. Derkus, C.C. Galeano, A. Hasan, Y. Wu, J. Ramis, L. Buttery, J.I. Dawson, M. D'Este, R.O.C. Oreffo, D. Eglin, H. Sun, A. Mata, Growth-factor free multicomponent nanocomposite hydrogels that stimulate bone formation, Adv. Func. Mater. (2020) 1906205.

[53] C. Yang, B. Han, C. Cao, D. Yang, X. Qu, X. Wang, An injectable double-network hydrogel for the co-culture of vascular endothelial cells and bone marrow mesenchymal stem cells for simultaneously enhancing vascularization and osteogenesis, J. Mater. Chem. B 6 (2018) 7811.

[54] S. Chen, A. Zhou, B. He, W. Zhao, X. Chen, and D. Jiang, Designer D-form selfassembling peptide scaffolds promote the proliferation and migration of rat bone marrowderived mesenchymal stem cells, Int. J. Mol. Med. 40 (2017) 679.

[55] M. Vishnu-Priya, A. Sivshanmugam, A.R. Boccaccini, O.M. Goudouri, W. Sun, N. Hwang, S. Deepthi, S.V. Nair, R. Jayakumar, Injectable osteogenic and angiogenic nanocomposite hydrogels for irregular bone defects, Biomed. Mater. 15;11(3) (2016) 035017.

[56] V. Lukasova, M. Buzgo, V. Sovkova, J. Dankova, M. Rampichova, E. Amler, Osteogenic differentiation of 3D cultured mesenchymal stem cells induced by bioactive peptides, Cell Prolif. 50(4) (2017) 12357.

[57] B. Byambaa, N. Annab, K. Yue, G. Trujillo-de Santiago, M.M. Alvarez, W. Jia, M. Kazemzadeh-Narbat, S.R. Shin, A. Tamayol, and A. Khademhosseini, Bioprinted osteogenic and vasculogenic patterns for engineering 3D bone tissue Adv. Healthcare Mater. 6(16) (2017) 1700015 .

[58] M. Kazemzadeh-Narbat, J. Rouwkema, N. Annabi, H. Cheng, M. Ghaderi, B.H. Cha, M. Aparnathi, A. Khalilpour, B. Byambaa, E. Jabbari, A. Tamayol, A. Khademhosseini, Engineering Photocrosslinkable Bicomponent Hydrogel Constructs for Creating 3D Vascularized Bone, Adv. Healthcare Mater. 6 (2017) 1601122.

[59] H. Yukawa, K. Suzuki, K. Aoki, T. Arimoto, T. Yasui, N. Kaji, T. Ishikawa, T. Ochiya, Y. Baba, Imaging of angiogenesis of human umbilical vein endothelial cells by uptake of exosomes secreted from hepatocellular carcinoma cells, Sci. Rep. 8 (2018) 6765.

[60] A.M. Goodwin, In vitro assays of angiogenesis for assessment of angiogenic and antiangiogenic agents, Microvasc. Res. 74 (2007) 172.

[61] M.P. Hendricks, K. Sato, L.C. Palmer, S.I. Stupp, Supramolecular assembly of peptide amphiphiles, Acc. Chem. Res. 50(10) (2017) 2440. 


\section{FIGURE CAPTIONS}

Figure 1. Synthesis and characterization of PAs. a) Molecular structures of each component namely HA-Tyr and PAs. b) Illustrations of 2D and 3D ECM-like co-assembled multicomponent hydrogels. Numbers in parenthesis depict the order in which the components were added during gel formation. c) CD spectra indicating the $\beta$-sheet formation and coassembly process for the PA systems at $0.1 \%$ concentration. d) TEM images of Pas (Blue arrows indicate high aspect ratio nanofibers). e) SEM images of (i) 6\% HA-Tyr hydrogel, (ii) HA-Tyr/RGDS-PA/Osteo-PA-Angio-PA hydrogel using 6\% HA-Tyr and 2\% PAs, and (iii) co-assembled PAs (2\%) without HA-Tyr.

Figure 2. Multicomponent hydrogel promotes hAMSC viability, adhesion, and spreading in 2D. a) 2D cell viability, adhesion, and spreading for calcein stained hAMSCs in different conditions. (i) Cell adhesion on HA-Tyr/RGDS-PA/Osteo-PA/Angio-PA at different time points. (ii) Enhanced cell adhesion on RGDS-PA-containing hydrogels. (iii) Effect of PA concentration on cell adhesion. b) Cell proliferation in 2D on HA-Tyr with and without RGDS-PA/Osteo-PA/Angio-PA at different concentrations. ( $*$ indicates $\mathrm{p}<0.05 ; * *$ indicates $\mathrm{p}>0.05)$.

Figure 3. Multicomponent hydrogel promotes hAMSC viability, adhesion, and spreading in 3D. a) Cell viability, adhesion, and spreading in 3D HA-Tyr hydrogels with and without RGDS-PA/Osteo-PA/Angio-PA at different time points. b) Cell proliferation in 3D HA-Tyr hydrogels with and without RGDS-PA/Osteo-PA/Angio-PA. (* indicates $\mathrm{p}<0.05$; ** indicates $\mathrm{p}>0.05$ ).

Figure 4. Multicomponent hydrogel supports vascular tubule formation in 2D and 3D. a) 2D angiogenesis assay with 40K HUVECs per hydrogel after 3 days on (i) HA-Tyr, (ii) HATyr/RGDS-PA, (iii) HA-Tyr/RGDS-PA/Angio-PA (0.5\% Angio-PA), (iv) HA-Tyr/RGDSPA/Angio-PA (1\% Angio-PA), (v) Matrigel (1:4 dilutions, +VEGF), and (vi) TCP. b) 2D immunofluorescence images of the angiogenic markers CD31 and $\alpha \mathrm{SMA}$ at 5-days incubation of 40K HUVECs on HA-Tyr/RGDS-PA/Angio-PA and TCP (negative control). c) RT-qPCR quantification of CD31 and VEGF genes expressed by HUVECs growing on HA-Tyr/RGDS-PA/Angio-PA and HA-Tyr/RGDS-PA (negative control). d) 3D angiogenesis assay for cells growing within HA-Tyr (negative control), HA-Tyr/RGDSPA/Angio-PA, and Matrigel (positive control, +VEGF) at 5-days incubation using $40 \mathrm{~K}$ HUVECs per hydrogel. S2D: Stacked multilayer 2D image. ${ }^{*} \mathrm{p}<0.05$.

Figure 5. Multicomponent hydrogel supports osteogenic differentiation in 2D and 3D. a) Characterization of osteogenic differentiation of hAMSCs seeded on HA-Tyr/RGDSPA/Osteo-PA (in GM), Matrigel (in OIM) (positive control), and HA-Tyr/RGDS-PA (in GM) (negative control) by immunofluorescence staining for Osn, ALP, and Runx2 at day 14 of culture. RT-qPCR quantification of Runx2 expressions for 2D (b) and 3D (c) hydrogels in different conditions. ${ }^{*} \mathrm{p}<0.05, * * \mathrm{p}<0.01$, ns: not significant.

Figure 6. Multicomponent hydrogel induces bone-like construct formation. a) 2D coculture at 5 days of pre-stained hAMSCs and HUVECs on HA-Tyr and HA-Tyr/RGDSPA/Osteo-PA/Angio-PA with different cell ratios including (i) hAMSCs and HUVECs (10K:10K) on HA-Tyr, (ii) hAMSCs and HUVECs (10K:10K) on HA-Tyr/RGDSPA/Osteo-PA/Angio-PA, (iii) hAMSCs and HUVECs (5K:20K) on HA-Tyr/RGDSPA/Osteo-PA/Angio-PA, and (iv) hAMSCs and HUVECs (10K:40K) on HA-Tyr/RGDSPA/Osteo-PA/Angio-PA. hAMSCs were stained with DiO (green) while HUVECs were stained with Dil (red) using Vybrant ${ }^{\mathrm{TM}}$ Multicolor Cell-Labelling Kit, Thermo. b) Images of 
co-cultures of hAMSCs and HUVECs $(10 \mathrm{~K}: 40 \mathrm{~K})$ in 3D at 5 days of culture including 3D (top) and stacked multilayer 2D (bottom) images. Gene expression related to Runx2, CD31, and VEGF indicating a co-differentiation of cells towards the development of a vascularized bone-like construct on both c-e) 2D hydrogels and f-h) 3D hydrogels after 14 days of coculture. $* \mathrm{p}<0.05, * * \mathrm{p}<0.01$, ns: not significant. 


\section{FIGURES}

a

$\mathrm{C}_{15} \mathrm{H}_{31}$ CO-VVVAAAKKKSVVYGLR (Angio-PA)

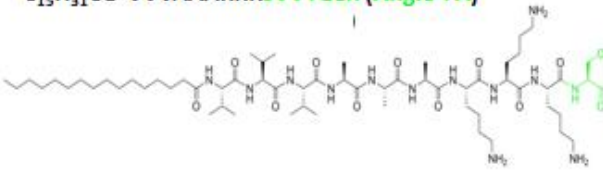

$\mathrm{C}_{15} \mathrm{H}_{31}$ CO-VVVAAAEEERGDS (RGDS-PA)

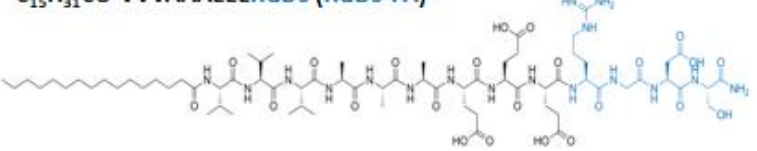

$\mathrm{C}_{15} \mathrm{H}_{31}$ CO-VVVAAADGEA (Osteo-PA)
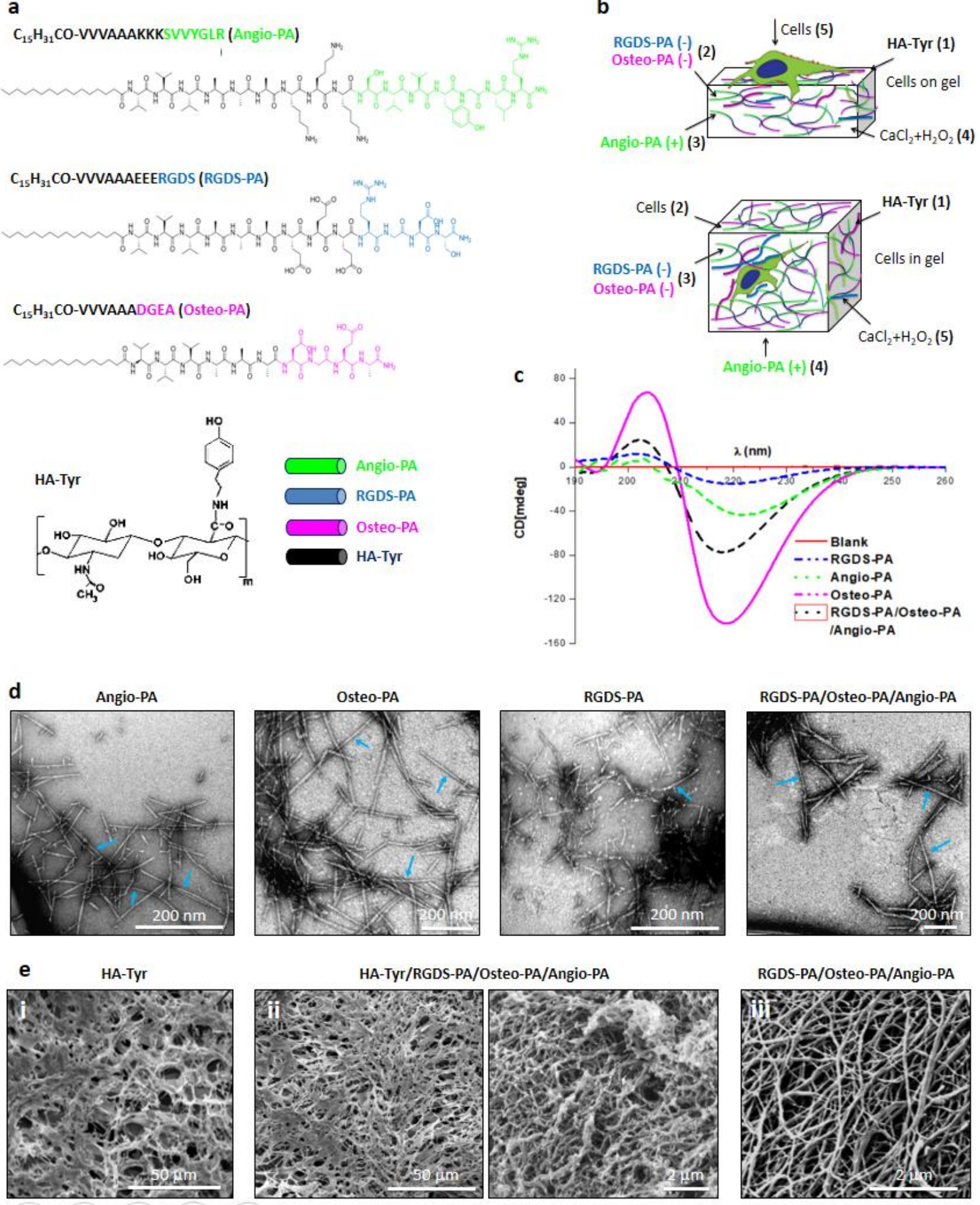

RGDS-PA/Osteo-PA/Angio-PA

Figure 1

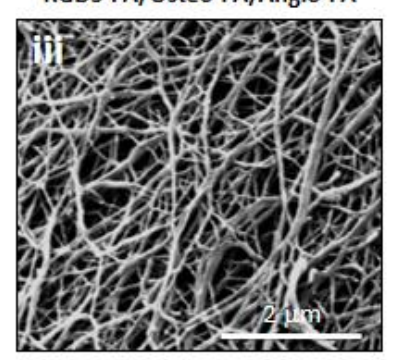




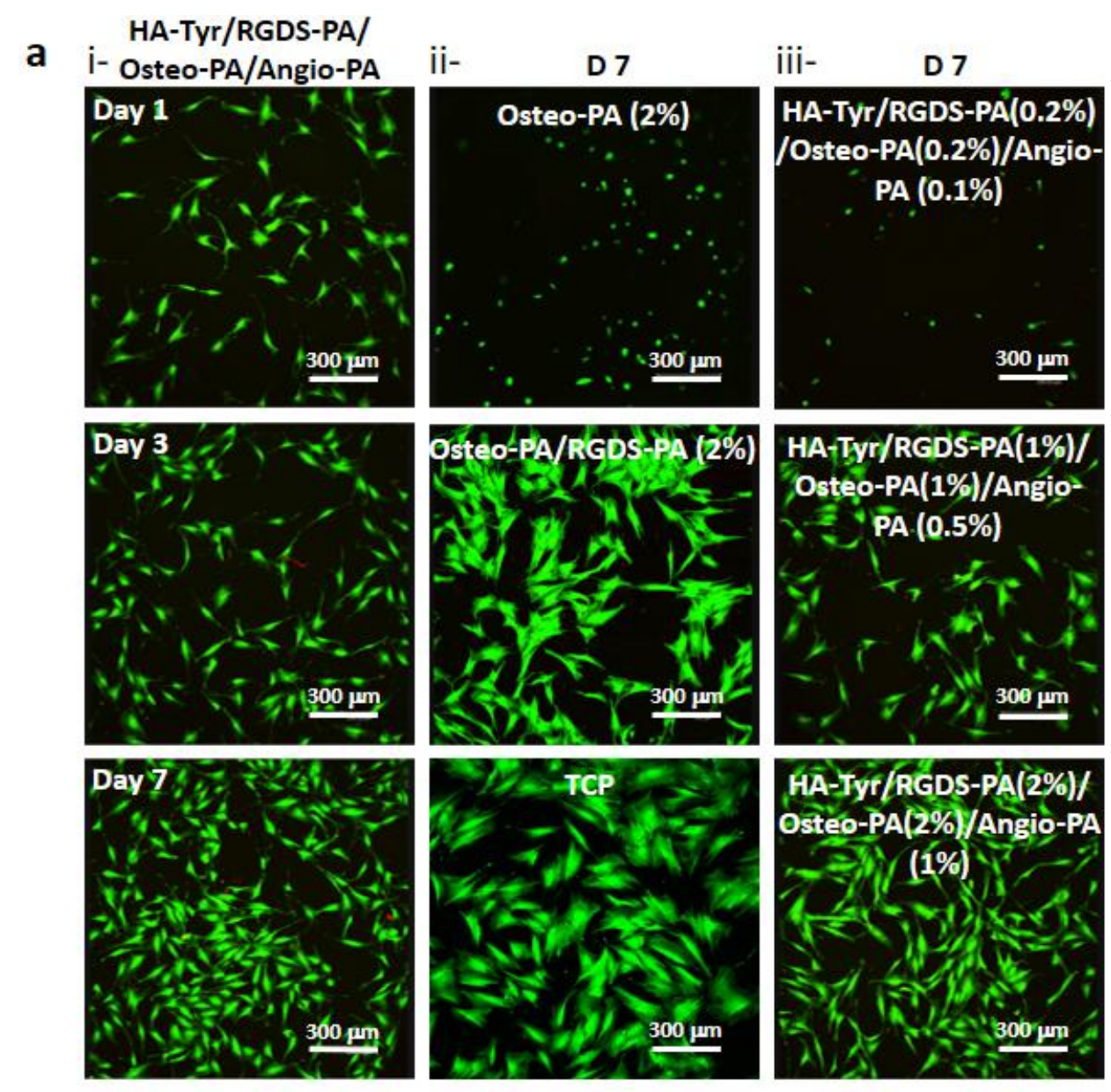

b

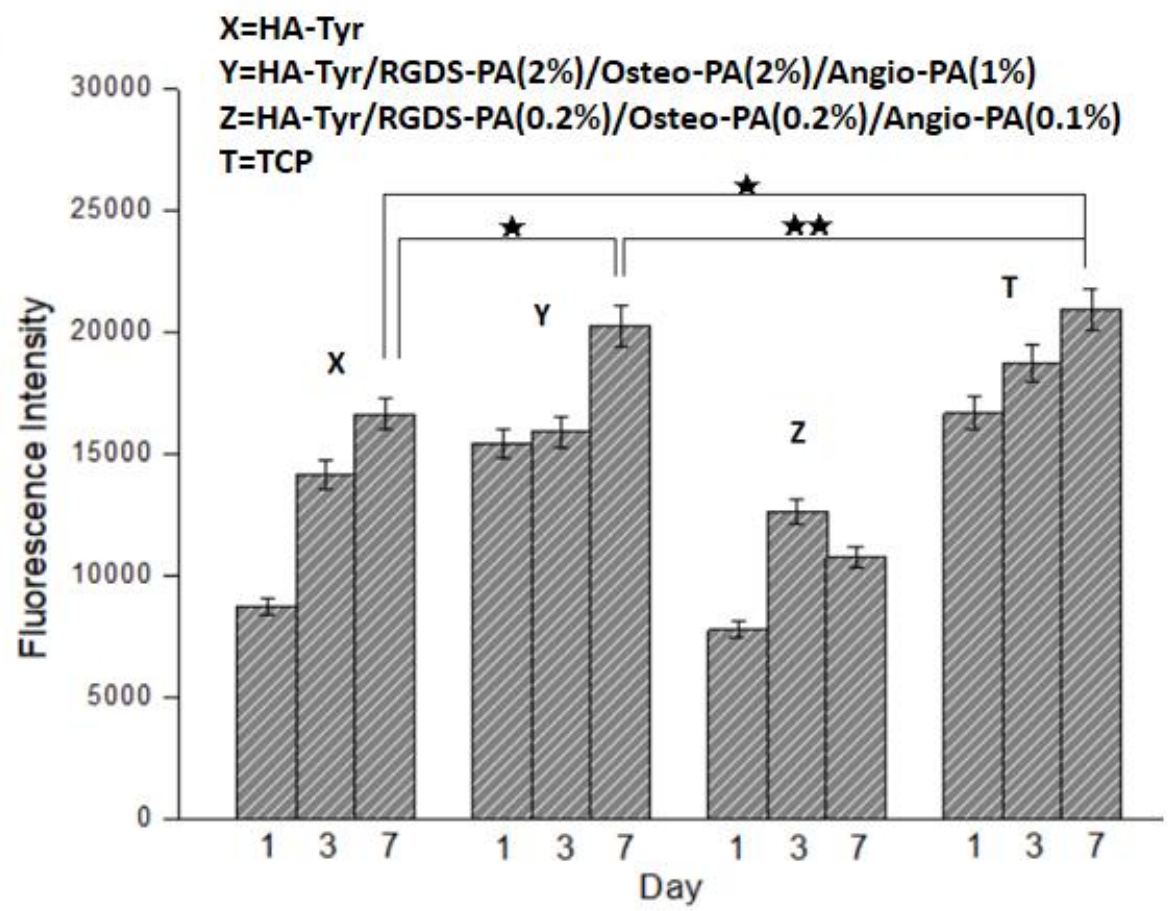

Figure 2 


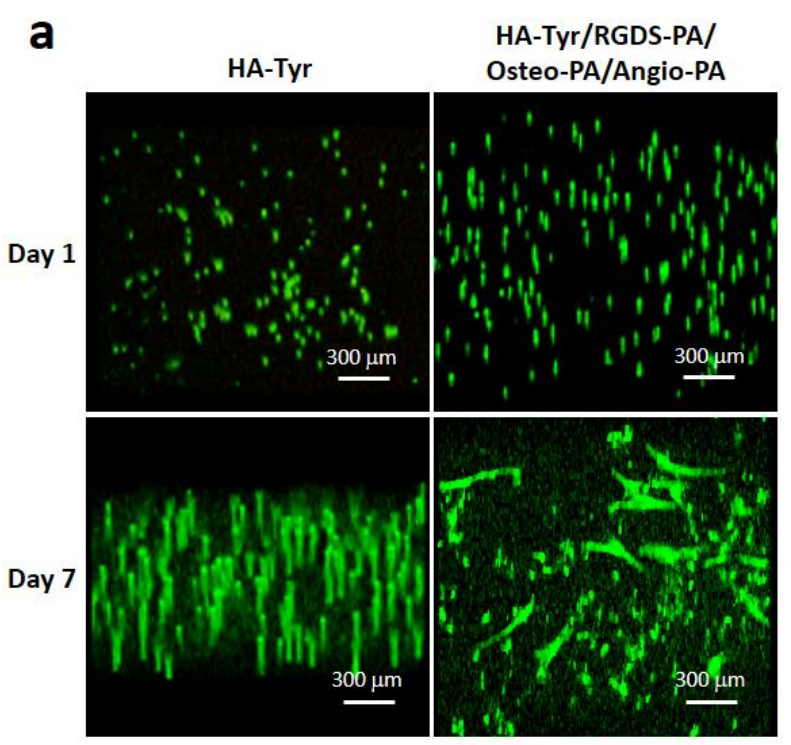

\section{b}

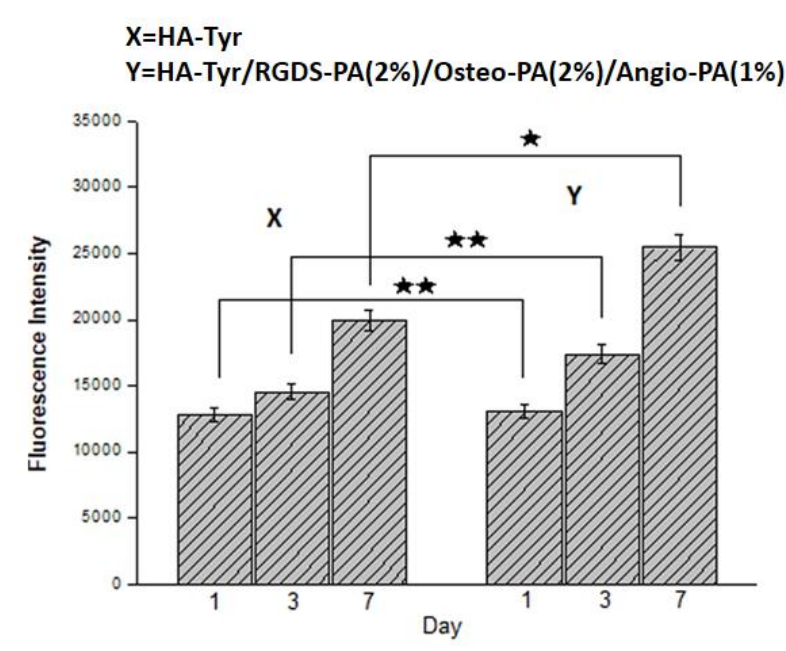

Figure 3 
a
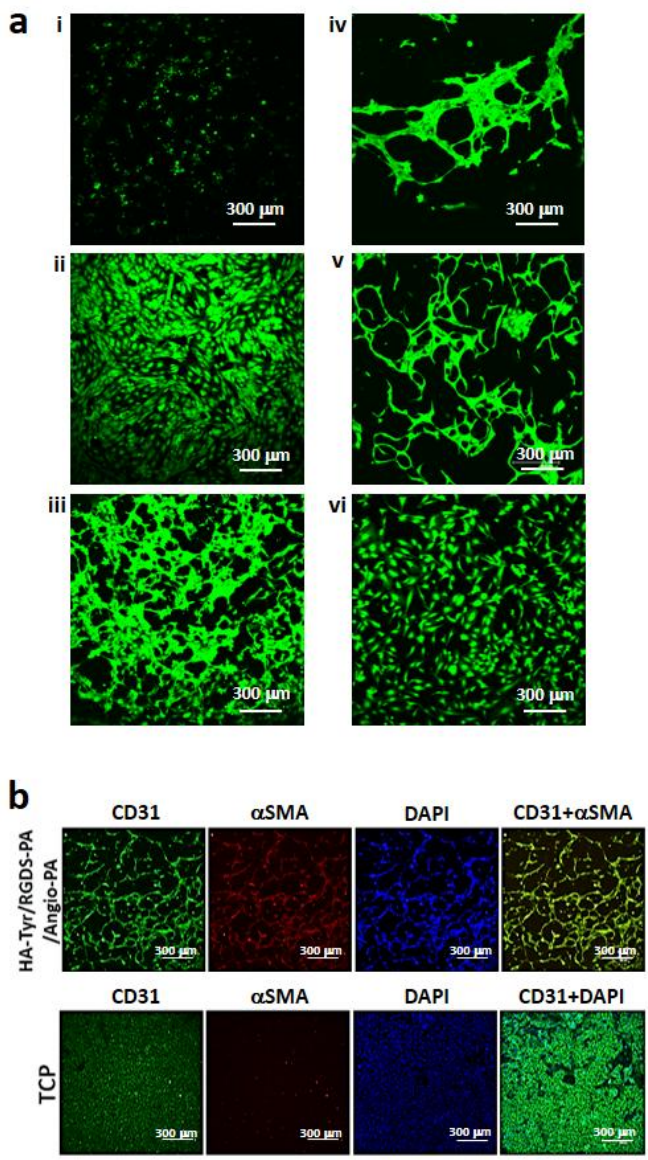
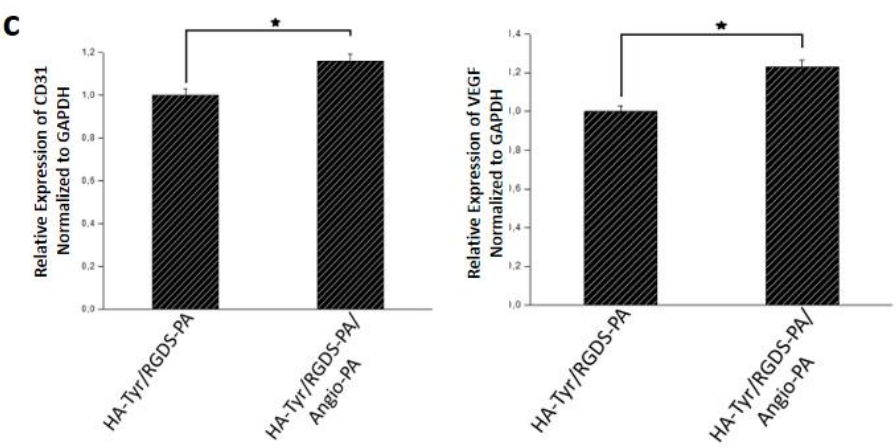

d

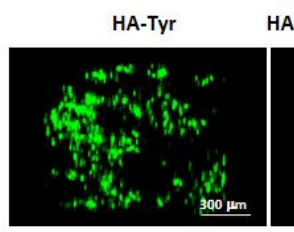

HA-Tyr/RGDS-PA/Angio-PA
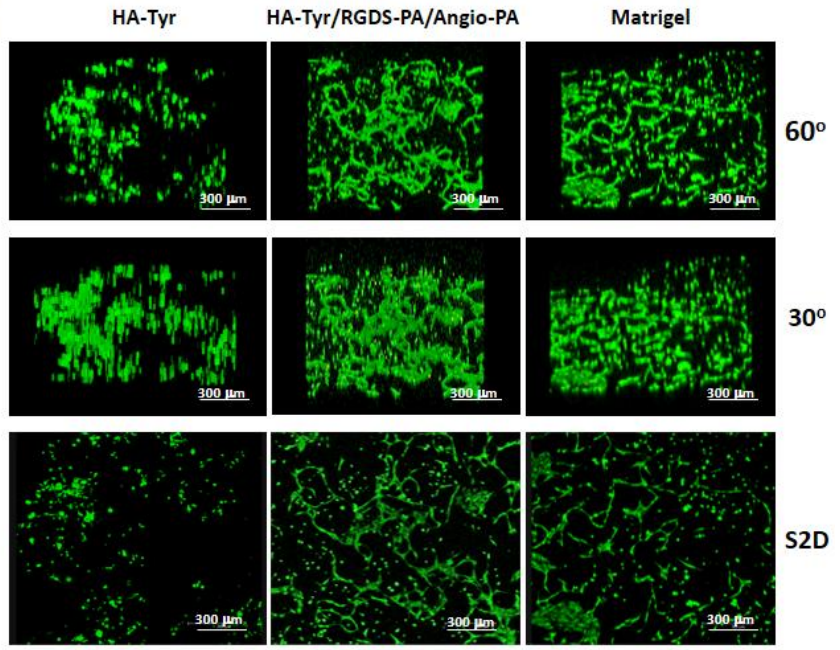

Figure 4 
a

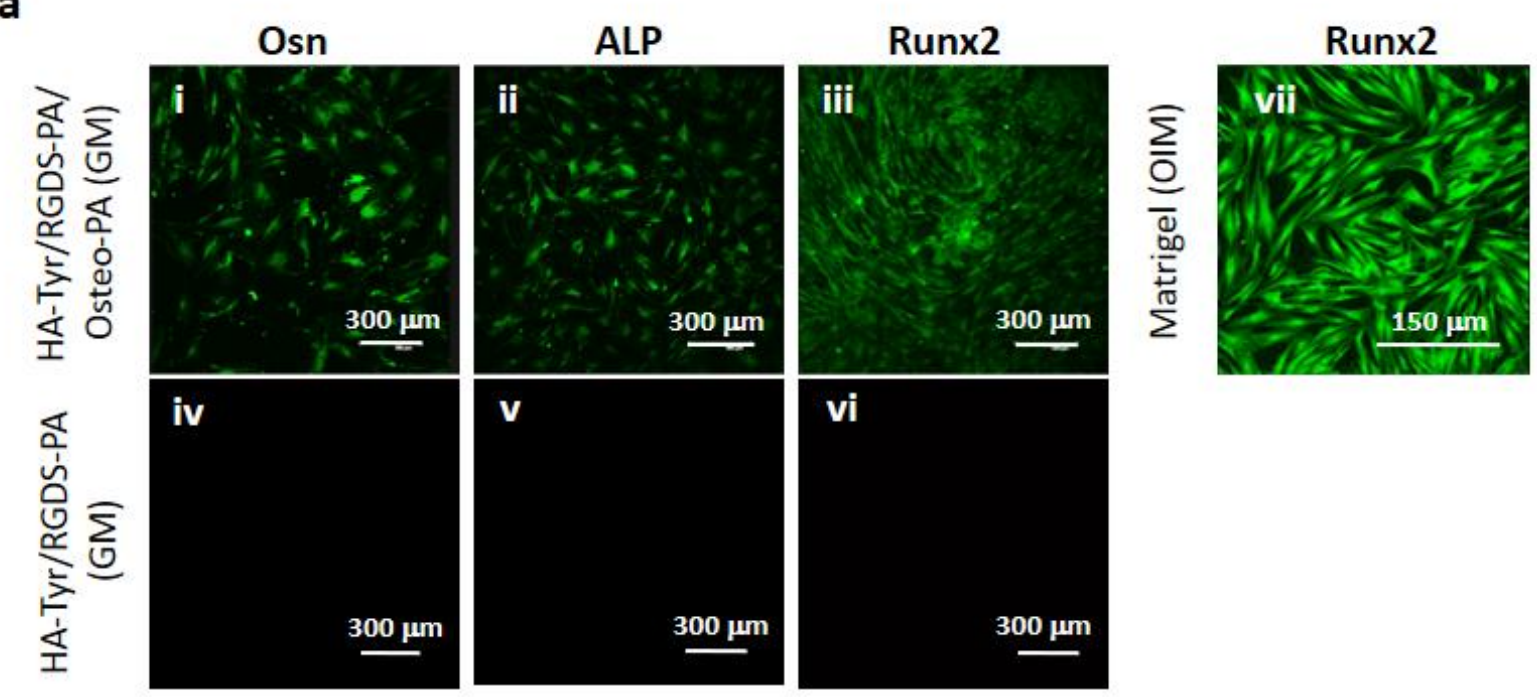

b

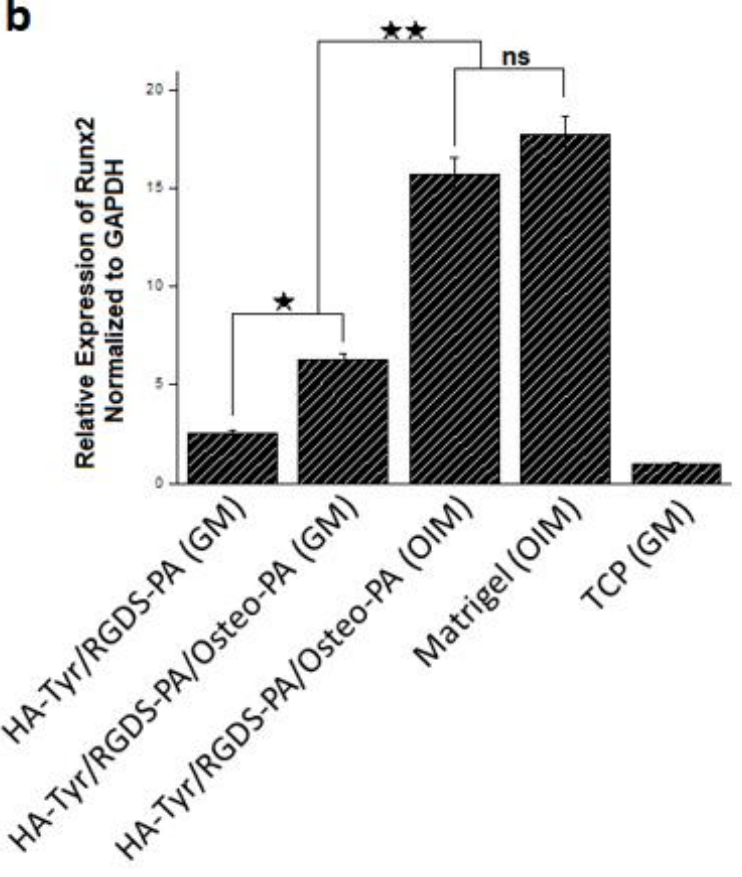

C

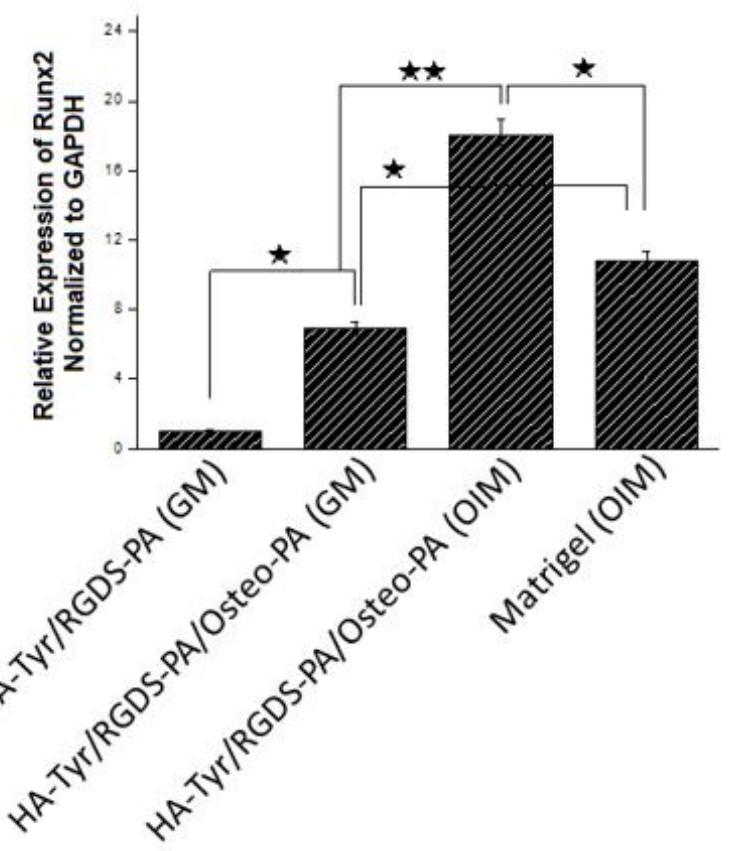

Figure 5 
a

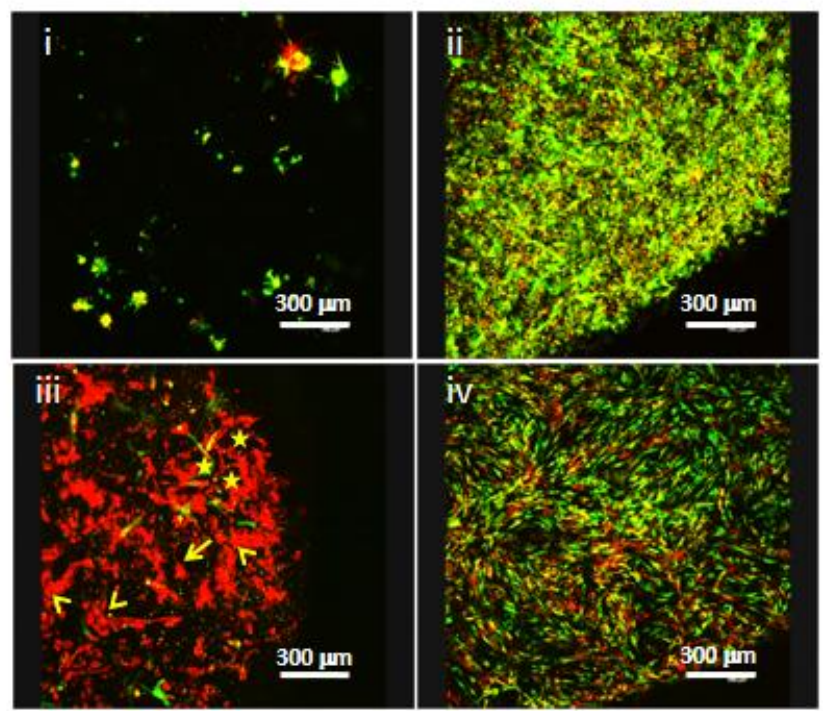

C

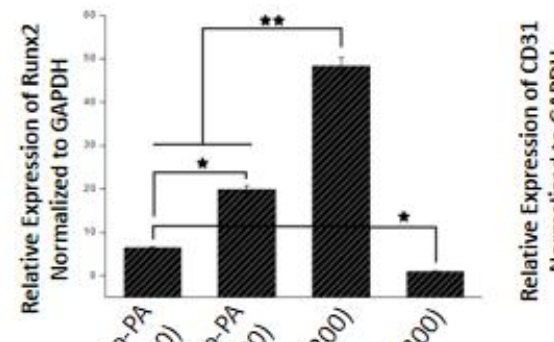

b
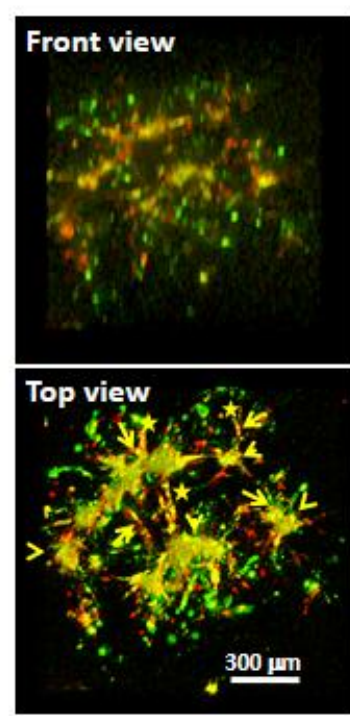

e

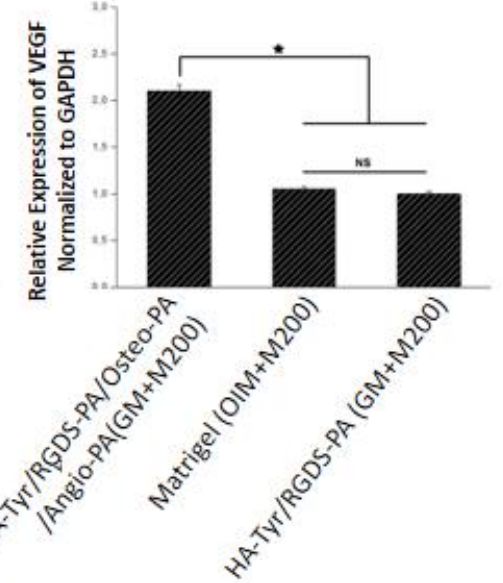

h

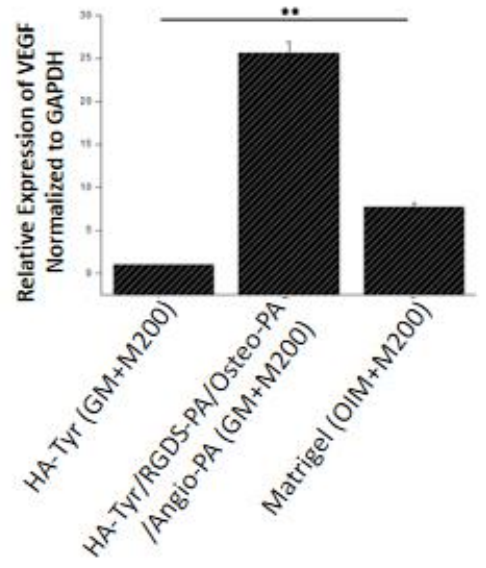

Figure 6 
Click here to download Supplementary Material: Derkus et al Resubmission - Supplementary Information.docx 


\section{Declaration of interests}

$\bigotimes$ The authors declare that they have no known competing financial interests or personal relationships that could have appeared to influence the work reported in this paper.

$\square$ The authors declare the following financial interests/personal relationships which may be considered as potential competing interests: 